TRANSACTIONS OF THE

AMERICAN MATHEMATICAL SOCIETY

Volume 353, Number 1, Pages 209-245

S 0002-9947(00)02597-6

Article electronically published on July 12, 2000

\title{
ON THE INVARIANT FACES ASSOCIATED WITH A CONE-PRESERVING MAP
}

\author{
BIT-SHUN TAM AND HANS SCHNEIDER
}

\begin{abstract}
For an $n \times n$ nonnegative matrix $P$, an isomorphism is obtained between the lattice of initial subsets (of $\{1, \cdots, n\}$ ) for $P$ and the lattice of $P$-invariant faces of the nonnegative orthant $\mathbb{R}_{+}^{n}$. Motivated by this isomorphism, we generalize some of the known combinatorial spectral results on a nonnegative matrix that are given in terms of its classes to results for a conepreserving map on a polyhedral cone, formulated in terms of its invariant faces. In particular, we obtain the following extension of the famous Rothblum index theorem for a nonnegative matrix: If $A$ leaves invariant a polyhedral cone $K$, then for each distinguished eigenvalue $\lambda$ of $A$ for $K$, there is a chain of $m_{\lambda}$ distinct $A$-invariant join-irreducible faces of $K$, each containing in its relative interior a generalized eigenvector of $A$ corresponding to $\lambda$ (referred to as semidistinguished $A$-invariant faces associated with $\lambda$ ), where $m_{\lambda}$ is the maximal order of distinguished generalized eigenvectors of $A$ corresponding to $\lambda$, but there is no such chain with more than $m_{\lambda}$ members. We introduce the important new concepts of semi-distinguished $A$-invariant faces, and of spectral pairs of faces associated with a cone-preserving map, and obtain several properties of a cone-preserving map that mostly involve these two concepts, when the underlying cone is polyhedral, perfect, or strictly convex and/or smooth, or is the cone of all real polynomials of degree not exceeding $n$ that are nonnegative on a closed interval. Plentiful illustrative examples are provided. Some open problems are posed at the end.
\end{abstract}

\section{INTRODUCTION}

This is the fourth of a sequence of papers studying the classical Perron-Frobenius theory of a nonnegative matrix and its generalizations to cone-preserving maps in the finite-dimensional setting from a cone-theoretic viewpoint, which is a geometric approach as opposed to the traditional graph-theoretic (combinatorial) approaches (cf., for instance, [Rot and [J-V3]). The first three papers in this sequence are T-W], Tam3 and [T-S1]. Different fundamental aspects of a cone-preserving map are considered, one in each paper of the sequence - the Collatz-Wielandt sets

Received by the editors October 31, 1997 and, in revised form, March 11, 1999.

2000 Mathematics Subject Classification. Primary 15A48; Secondary 47B65, 47A25, 46B42.

Key words and phrases. Cone-preserving map, nonnegative matrix, polyhedral cone, perfect cone, strictly convex smooth cone, spectral pair of a vector, spectral pair of a face, Perron-Schaefer condition, initial subset, semi-distinguished class, semi-distinguished invariant face, distinguished generalized eigenvector, chain of invariant faces.

Research of the first author partially supported by the National Science Council of the Republic of China grant NSC 86-2115-M-032-002; the second author's research partially supported by NSF grants DMS-9123318 and DMS-9424346.

(C)2000 American Mathematical Society 
(or numbers) in [T-W], the distinguished eigenvalues in [Tam3], the core in [T-S1], and the invariant faces in this paper.

In $\mathrm{T}-\mathrm{S} 1$, the preceding paper of our sequence, we studied the core of a conepreserving map $A$, i.e. the convex cone $\operatorname{core}_{K}(A):=\bigcap_{i=1}^{\infty} A^{i} K$. We were able to characterize the $K$-irreducibility or $K$-primitivity of $A$ in terms of its core, and also to obtain many interesting results that give connections between the core, the peripheral spectrum, the Perron-Schaefer condition and the distinguished $A$ invariant faces of $K$. (We shall define some of these terms later on.) Nevertheless, the results we obtained directly or indirectly suggest that the set $\operatorname{core}_{K}(A)$ does not capture all the important information about the spectral properties of $A$. In particular, if $\operatorname{core}_{K}(A)$ is a polyhedral cone, then it does not contain any distinguished generalized eigenvectors of $A$ other than eigenvectors. Thus, the index of the spectral radius of $A$ cannot be determined from a knowledge of its core. On the other hand, in the nonnegative matrix case, as is known, the index of the spectral radius or of a distinguished eigenvalue can be described in terms of its (equivalence) classes (of communicating states). Moreover, we found, in a preliminary study, a close connection between the classes of a nonnegative matrix and its associated invariant faces (of the corresponding nonnegative orthant). This motivates our present study of the invariant faces associated with a cone-preserving map.

Here we would like to stress the differences between our work and that of others on positive (linear) operators. So far, the deepest results in the theory of positive operators are mostly obtained under the assumption that the positive operator under consideration is irreducible or the underlying space is a nice space, such as a Lebesgue space or, more generally, a Banach lattice. Furthermore, many of these deep results have no counterparts in the finite-dimensional setting. A look at the monographs [MN2], Scha] or the recent survey paper by Dodds [Dod] will give the reader some ideas. (See also Zer for an earlier survey.) Over the past two decades, progress in a large part of the theory has followed three principal themes, namely, the kernel representation theorem of Bukhvalov (1974), the compact majorization theorem of Dodds-Fremlin (1979) and the spectral radius theorem of de Pagter (1986). These results are given in the settings of the familiar Lebesgue spaces or of a Banach lattice and, as can be readily seen none of them have a finite-dimensional counterpart, as their conclusions (that a certain operator is compact or is a kernel operator) are trivially satisfied when the underlying space is of finite dimension.

Nevertheless, the recent development of the combinatorial spectral theory of nonnegative matrices (applied to the reducible case which is much more complex than the irreducible case, see for instance [Schn3]) has had some impact on the theory of positive operators. For instance, many of the graph-theoretic ideas (such as the concepts of classes, the notion of accessibility between states or classes) or of the combinatorial spectral results (such as the nonnegative basis theorem and the Rothblum index theorem) of a nonnegative matrix, implicit in the early work of Schneider ([Schn1]) and formulated first by Rothblum [Rot], have already been extended, first to the setting of an eventually compact linear integral operator on $L^{p}(\Omega, \mu), 1 \leq p<\infty$, with nonnegative kernel (see [Nel], Vic1, Vic2], JJ-V1]), and then to the setting of a positive, eventually compact linear operator on a Banach lattice having order continuous norm (see [J-V2, J-V3] $)$. Their treatment, which employs mainly functional-analytic methods, is made possible by a decomposition of the positive operator under consideration in terms of certain closed ideals of the underlying space in a form which directly generalizes the Frobenius normal form 
of a nonnegative reducible matrix. In this work, we are also generalizing theorems on reducible nonnegative matrices. However, we shall not generalize the Frobenius normal form; indeed, we do not expect there is a natural generalization for a conepreserving map when the underlying cone is a general proper cone. Our focus is on the invariant faces, and our results are geometric and provide new information in finite dimension. Moreover, we do not confine ourselves to a lattice-ordered cone, which in the finite-dimensional setting reduces to the classical nonnegative matrix case.

We now describe the contents of this paper in some detail. Some necessary definitions and preliminary results are collected in Section 2.

For a nonnegative matrix, we have the known concepts of basic classes, distinguished classes, initial classes, etc. In order to find for a cone-preserving map the appropriate analogs of these concepts, in Section 3 we begin our investigation by studying the lattice of $P$-invariant faces of $\mathbb{R}_{+}^{n}$ (the nonnegative orthant of $\mathbb{R}^{n}$ ) associated with an $n \times n$ nonnegative matrix $P$. We introduce the combinatorial concept of an initial subset (of $\{1, \cdots, n\}$ ) for $P$. An isomorphism is found between the lattice of initial subsets for $P$ and the lattice of $P$-invariant faces of $\mathbb{R}_{+}^{n}$, thus relating a combinatorial concept to a geometric concept via algebraic structures. In particular, under this isomorphism a $P$-invariant join-irreducible face which contains in its relative interior a generalized eigenvector of $P$ corresponding to $\rho(P)$, the spectral radius of $P$, corresponds to an initial subset determined by a basic class. This isomorphism motivates the subsequent attempts in this paper to generalize many of the known combinatorial spectral results on a nonnegative matrix that are given in terms of its classes to results for a cone-preserving map, formulated in terms of its invariant faces. In the course of establishing this isomorphism, we reprove the well-known Frobenius -Victory theorem, introduce the concept of a semi-distinguished class for a nonnegative matrix, reformulate the known nonnegative basis theorem for the generalized eigenspace corresponding to a distinguished eigenvalue of a nonnegative matrix and offer a cone-theoretic proof.

The following are two of the well-known results on the combinatorial spectral theory of a nonnegative matrix that we wish to extend in this paper.

Theorem A (Rothblum index theorem [Rot, Theorem 3.1(2)]). If $P$ is a nonnegative matrix, then there always exists a chain of $\nu$ basic classes of $P$, where $\nu$ is the index of $\rho(P)$ as an eigenvalue of $P$, but there is no chain with more than $\nu$ basic classes.

Theorem B (Schn1, 1, Theorem 5]). If $P$ is a nonnegative matrix, then the Jordan canonical form of $P$ contains only one block corresponding to $\rho(P)$ (that is, $\operatorname{dim} \mathcal{N}(\rho(P) I-P)=1$ ) if and only if any two basic classes of $P$ are comparable (with respect to the accessibility relation).

In our investigation, we find it useful to introduce the concept of the spectral pair of a face relative to a cone-preserving map. We do this in Section 4. Given an $n \times n$ complex matrix $A$, for any vector $x \in \mathbb{C}^{n}$, we assign to it an ordered pair $\left(\rho_{x}(A), \operatorname{ord}_{A}(x)\right)$, where $\rho_{x}(A)$ is the local spectral radius of $A$ at $x$ and $\operatorname{ord}_{A}(x)$ is the order of $x$ relative to $A$, a new concept which we introduce as an extension of the usual concept of the order of a generalized eigenvector. We denote this ordered pair by $\operatorname{sp}_{A}(x)$ and refer to it as the spectral pair of $x$ relative to $A$. If $A$ leaves invariant

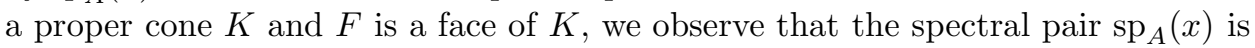
independent of the choice of $x$ from the relative interior of $F$. This constant value 
is denoted by $\operatorname{sp}_{A}(F)$, and will be referred to as the spectral pair of $F$ relative to $A$. Fundamental properties of spectral pairs of faces (or of vectors in the underlying cone) relative to a cone-preserving map are derived. One consequence is that we obtain a class of $A$-invariant faces of $K$ given in terms of spectral pairs and the lexicographic ordering defined between ordered pairs of real numbers, which extends and refines in the finite-dimensional case the class of invariant ideals discovered by Meyer-Nieberg [MN1] for a positive linear operator defined on a Banach lattice. We also prove several equivalent conditions for a distinguished $A$-invariant face, a concept which we introduced in our preceding paper [T-S1] as an extension of the concept of a distinguished class of a nonnegative matrix. Then, as a natural extension of the concept of a semi-distinguished class, we call an $A$-invariant face semi-distinguished if it is $A$-invariant join-irreducible and contains in its relative interior a generalized eigenvector of $A$.

In Section 5 , in terms of semi-distinguished $A$-invariant faces we formulate and establish a natural extension of the Rothblum index theorem in the setting of a linear mapping preserving a polyhedral cone. We also give examples which show that in the nonpolyhedral case the result is no longer true.

We take a closer look at semi-distinguished $A$-invariant faces in Section 6 . Further properties of invariant faces (mostly involving spectral pairs, semi-distinguished invariant faces, or distinguished generalized eigenvectors) associated with a linear mapping preserving a polyhedral cone are obtained. It is proved that if $A$ leaves invariant a polyhedral cone $K$, then a nonzero $A$-invariant face $F$ of $K$ is semidistinguished if and only if for any $A$-invariant face $G$ properly included in $F$, we have, $\operatorname{sp}_{A}(G) \prec \operatorname{sp}_{A}(F)$, where $\prec$ denotes the strict lexicographic ordering between ordered pairs of real numbers. The nonpolyhedral case is also examined in detail. It appears that whether a cone-preserving map shares some of the properties of a cone-preserving map on a polyhedral cone depends much on the geometry of the underlying cone. In particular, we prove that if $A$ leaves invariant a proper cone $K$ which has the property that the dual cone of each of its faces is a facially exposed cone, then the above-mentioned characterization of semi-distinguished $A$-invariant faces in terms of the lexicographic ordering still holds.

In Section 7 we obtain an extension and a refinement of Theorem B in the setting of a linear mapping preserving a polyhedral cone. The situation for the nonpolyhedral case is also investigated carefully.

We give some multi-purpose examples in Section 8, and pose some open questions in Section 9, the final section of our paper.

Applications of our theory to the study of solvability of linear equations over cones and Collatz-Wielandt numbers will be given in a forthcoming paper [T-S2].

Before we end this section, we would like to point out that in this paper our proofs of theorems on a linear mapping preserving a polyhedral cone rely much on the fact that if $A$ leaves invariant a polyhedral cone $K$, then $K$ contains generalized eigenvectors of $A$ of all possible orders between 1 and $\nu$, where $\nu$ is the index of $\rho(A)$ as an eigenvalue of $A$. The latter fact follows from [Tam3, Theorem 7.5(ii)], which asserts that there always exists a vector $x$ in $K$ (a polyhedral cone) such that $(A-\rho(A) I)^{\nu} x=\mathbf{0}$, and $(A-\rho(A) I)^{i} x$ are nonzero vectors of $K$ for $i=0, \cdots, \nu-1$. This last fact is, in turn, proved in Tam3, using the minimal generating matrix of a polyhedral cone as a tool and depending on the corresponding known result for a nonnegative matrix. However, in our next paper Tam4, we shall offer an 
analytic and cone-theoretic proof of a result stronger than this last fact, without assuming the corresponding result for a nonnegative matrix. Thus, our treatment can be kept independent of the known results on a nonnegative matrix.

\section{Preliminaries}

We shall restrict our attention to finite-dimensional vector spaces. A familiarity with convex cones, cone-preserving maps, and graph-theoretic properties of nonnegative matrices is assumed. (For references, see [Bar2], B-P] or [Schn3].) The terminology and notation of the previous papers in this sequence are adopted here. For convenience, we collect some of them below, together with some additional new definitions and notation.

Unless specified otherwise, all matrices considered in this paper are square. We always use $K$ to denote a proper (i.e. closed, full, pointed convex) cone of $\mathbb{R}^{n}$, and $\pi(K)$ to denote the set of all $n \times n$ real matrices $A$ that satisfy $A K \subseteq K$. Elements of $\pi(K)$ are referred to as cone-preserving maps (or more commonly as positive-operators on $K$ ).

If $S \subseteq K$, we denote by $\Phi(S)$ the face of $K$ generated by $S$, that is, the intersection of all faces of $K$ including $S$. If $x \in K$, we write $\Phi(\{x\})$ simply as $\Phi(x)$. A vector $x \in K$ is called an extreme vector if either $x$ is the zero vector or $x$ is nonzero and $\Phi(x)=\{\lambda x: \lambda \geq 0\}$; in the latter case, the face $\Phi(x)$ is called an extreme ray. A proper cone $K$ is said to be polyhedral if it has finitely many extreme rays.

The set of all faces of $K$ is denoted by $\mathcal{F}(K)$. By a nontrivial face of $K$ we mean a face other than $K$ itself or the zero face $\{\mathbf{0}\}$. By the dual cone $K^{*}$ of $K$ we mean the proper cone $\left\{z \in \mathbb{R}^{n}:\langle z, x\rangle \geq 0\right.$ for all $\left.x \in K\right\}$, where $\langle z, x\rangle$ is the usual inner product of $\mathbb{R}^{n}$ between the vectors $z$ and $x$. We also use $d_{K}$ to denote the duality operator of $K$, i.e. the mapping from $\mathcal{F}(K)$ to $\mathcal{F}\left(K^{*}\right)$ given by $d_{K}(F)=(\operatorname{span} F)^{\perp} \bigcap K^{*}($ see [Tam2] $)$. A face $F$ of $K$ is said to be exposed if there exists a face $G$ of $K^{*}$ such that $F=d_{K^{*}}(G)$, or, equivalently, if $F=d_{K^{*}} \circ d_{K}(F)$. A cone $K$ is called facially exposed if each of its faces is exposed; or equivalently, $d_{K}$ is injective, or $d_{K^{*}}$ is surjective (see Tam2, Proposition 2.5 (a) and Corollary $2.6])$.

The set of all $n \times n$ complex matrices is denoted by $\mathcal{M}_{n}$. The range space, nullspace and the spectral radius of a matrix $A \in \mathcal{M}_{n}$ are denoted respectively by $\mathcal{R}(A), \mathcal{N}(A)$ and $\rho(A)$. If $A$ is a real matrix, its range space and null space are understood to be taken in the corresponding real spaces.

Eigenvalues of $A$ with modulus $\rho(A)$ are said to compose the peripheral spectrum of $A$.

For any $A \in \mathcal{M}_{n}$ and $b \in \mathbb{C}^{n}$, by the cyclic space (relative to $A$ ) generated by the vector $b$, denoted by $W_{b}$, we mean the subspace $\operatorname{span}\left\{b, A b, A^{2} b, \cdots\right\}$. (Again, when $A$ and $b$ are both real, the above linear span is taken in the real space.) If $\lambda$ is an eigenvalue of $A$, we denote by $\nu_{\lambda}(A)$ the index of $\lambda$ as an eigenvalue of $A$.

Let $A$ be an $n \times n$ real matrix. It is known that a necessary and sufficient condition for the existence of a proper cone $K$ of $\mathbb{R}^{n}$ such that $A \in \pi(K)$ is that, for each eigenvalue $\lambda$ in the peripheral spectrum of $A$, we have $\nu_{\lambda}(A) \leq \nu_{\rho(A)}(A)$. (Then clearly $\rho(A)$ is an eigenvalue of $A$.) This condition is now referred to as the Perron-Schaefer condition (see Schn2, the paragraph after Theorem 1.1]).

If $A \in \mathcal{M}_{n}$ and $x$ is a nonzero vector of $\mathbb{C}^{n}$ such that $(\lambda I-A)^{k} x=\mathbf{0}$ for some $\lambda \in \mathbb{C}$ and some positive integer $k$, then $x$ is called a generalized eigenvector of $A$ 
corresponding to (the eigenvalue) $\lambda$. The least such integer $k$ is called the order of $x$ as a generalized eigenvector of $A$, and is denoted by $\operatorname{ord}_{A}(x)$.

For any $A \in \mathcal{M}_{n}$ and $x \in \mathbb{C}^{n}$, we define the local spectral radius of $A$ at $x$, denoted by $\rho_{x}(A)$, as follows. If $x$ is the zero vector, take $\rho_{x}(A)$ to be 0 . If $x$ is a nonzero vector, write $x$ uniquely as a sum of generalized eigenvectors of $A$, say,

$$
x=x_{1}+\cdots+x_{k},
$$

where $k \geq 1$, and $x_{1}, \cdots, x_{k}$ are generalized eigenvectors of $A$ corresponding to distinct eigenvalues $\lambda_{1}, \cdots, \lambda_{k}$ respectively. Define $\rho_{x}(A)$ to be $\max _{1 \leq i \leq k}\left|\lambda_{i}\right|$. For other equivalent definitions, see [T-W, Theorem 2.2].

If $A \in \pi(K)$ and $x \in K$ is an eigenvector (respectively, generalized eigenvector), then $x$ is called a distinguished eigenvector (respectively, distinguished generalized eigenvector) of $A$ for $K$, and the corresponding eigenvalue is known as a distinguished eigenvalue of $A$ for $K$. When there is no danger of confusion, we simply use the terms distinguished eigenvector, distinguished generalized eigenvector and distinguished eigenvalue (of $A$ ). (For reference, see Tam3.) By an extremal distinguished eigenvector of $A$ corresponding to the (distinguished) eigenvalue $\lambda$, we mean a nonzero extreme vector of the cone $\mathcal{N}(\lambda I-A) \cap K$.

The nonnegative orthant of $\mathbb{R}^{n}$ is denoted by $\mathbb{R}_{+}^{n}$. It is clear that $\mathbb{R}_{+}^{n}$ is a proper cone of $\mathbb{R}^{n}$, and also that $\pi\left(\mathbb{R}_{+}^{n}\right)$ is the cone of all $n \times n$ nonnegative matrices. When we consider an $n \times n$ nonnegative matrix as a cone-preserving map, unless specified otherwise, we always mean that the underlying cone is $\mathbb{R}_{+}^{n}$.

A familiarity with the diagraph and classes of a nonnegative matrix is assumed. (For reference, see, for instance, [Schn3].) If $n$ is a positive integer, we denote by $\langle n\rangle$ the set $\{1,2, \cdots, n\}$. We always use $P$ to denote an $n \times n$ nonnegative matrix for some positive integer $n$. The subsets of $\langle n\rangle$ are usually denoted by capital Latin letters $I, S$, etc., except that we use small Greek letters $\alpha, \beta$, etc. to denote the classes of $P$. The accessibility relation is usually defined between the classes of $P$, but we also say $i$ has access to $j$ (where $i, j \in\langle n\rangle$ ) if there is a directed path in $G(P)$ (the digraph of $P$ ) from $i$ to $j$. Similarly, we also say $i$ has access to a subset of $\langle n\rangle$ with the obvious meaning. If $S, T \subseteq\langle n\rangle$, we denote by $P_{S T}$ the principal submatrix of $P$ with rows indexed by $S$ and columns indexed by $T$.

Sometimes we say a class $\alpha$ of $P$ is associated with $\lambda$. By that we mean $\lambda=$ $\rho\left(P_{\alpha \alpha}\right)$. A class $\alpha$ is said to be semi-distinguished if $\rho\left(P_{\beta \beta}\right) \leq \rho\left(P_{\alpha \alpha}\right)$ for any class $\beta$ which has access to $\alpha$ but is not equal to $\alpha$. If, in this definition, the weak inequality is replaced by strict inequality of the same type, we recover the usual definition of a distinguished class.

A subset $I$ of $\langle n\rangle$ is called an initial subset for $P$ if either $I$ is empty, or $I$ is nonempty and $P_{I^{\prime} I}=\mathbf{0}$, where $I^{\prime}=\langle n\rangle \backslash I$; equivalently, for every $j \in\langle n\rangle, I$ contains $j$ whenever $j$ has access to $I$. Clearly an initial subset for $P$ which is also a class of $P$ is simply an initial class in the usual sense. It is also not difficult to see that a nonempty subset $I$ of $\langle n\rangle$ is an initial subset for $P$ if and only if $I$ is the union of an initial collection of classes of $P$, where a nonempty collection of classes of $P$ is said to be initial if whenever it contains a class $\alpha$, it also contains all classes having access to $\alpha$. One can readily verify that the intersection and union of two initial subsets for $P$ are both initial subsets for $P$. Thus the collection of all initial subsets for $P$ forms a sublattice of $2^{\langle n\rangle}$, the lattice of all subsets of $\langle n\rangle$. We denote this sublattice by $\mathcal{I}$. 
It is known that $\mathcal{F}(K)$ forms a lattice under inclusion as the partial ordering, with meet and join given by $F \wedge G=F \cap G$ and $F \vee G=\Phi(F \cup G)$. If $A \in \pi(K)$, then a face $F$ of $K$ is said to be $A$-invariant if $A F \subseteq F$. Clearly, the meet of two $A$ invariant faces is $A$-invariant. The join of two $A$-invariant faces is also $A$-invariant; this is because, for any two such faces $F, G$, we have

$$
A(F \vee G)=A(\Phi(F \cup G)) \subseteq \Phi(A(F \cup G)) \subseteq \Phi(F \cup G)=F \vee G
$$

Thus, the set of all $A$-invariant faces of $K$, which we denote by $\mathcal{F}_{A}(K)$ (or simply by $\mathcal{F}_{A}$ if there is no danger of confusion), forms a sublattice of $\mathcal{F}(K)$. A face $F$ of $K$ is said to be $A$-invariant join-reducible if $F$ is $A$-invariant and is the join of two (and hence, of all) $A$-invariant faces of $K$ that are properly included in $F$; in other words, $F$ is join-reducible in the lattice $\mathcal{F}_{A}$ in the usual lattice-theoretic sense. (For definitions of lattice-theoretic terms, see $\mathrm{Bir}$.) An $A$-invariant face which is not $A$-invariant join-reducible is said to be A-invariant join-irreducible. According to our definition, the zero face is always $A$-invariant join-irreducible.

For any $F \in \mathcal{F}_{A}$, we denote by $\rho_{F}(A)$ (or simply by $\rho_{F}$ ) the spectral radius of the restriction map $\left.A\right|_{\text {span } F}$; then we also say $F$ is associated with $\rho_{F}$. A face $F$ is said to be a distinguished A-invariant face of $K$ (associated with $\lambda$ ) if $F$ is a nonzero $A$-invariant face of $K$ such that $\rho_{G}<\rho_{F}$ for any nonzero $A$-invariant face $G$ properly included in $F$ (and $\rho_{F}=\lambda$ ).

We shall need the following explicit characterization of the smallest $A$-invariant face that contains a given vector $x$ of $K$.

Lemma 2.1. Let $A \in \pi(K)$ and let $x \in K$. Then $\Phi\left((I+A)^{n-1} x\right)$ is the smallest $A$-invariant face of $K$ containing $x$.

Proof. For convenience, denote the vector $(I+A)^{n-1} x$ by $y$. Note that $y$ is a positive linear combination of the vectors $x, A x, \cdots, A^{n-1} x$ of $K$ and also that these vectors span $W_{x}$ (the cyclic space generated by $x$ ). So $y$ belongs to the relative interior of $W_{x} \cap K$. But clearly $A y \in W_{x} \cap K$, so there exists some $\alpha>0$ such that $\alpha y-A y \in W_{x} \cap K$, and hence $\alpha y \geq^{K} A y$. Thus, $\Phi(y)$ is an $A$-invariant face which, in addition, also contains $x$, as $y \geq{ }^{K} x$. If $F$ is an $A$-invariant face of $K$ that contains $x$, then $F$ must contain $A x, \cdots, A^{n-1} x$, and hence also $y$; thus $F \supseteq \Phi(y)$. This proves that $\Phi(y)$ is the smallest $A$-invariant face of $K$ that contains $x$.

\section{The INVARIANT FACES ASSOCIATED WITH A NONNEGATIVE MATRIX}

It is known that every face of $\mathbb{R}_{+}^{n}$ is of the form

$$
F_{I}=\left\{x \in \mathbb{R}_{+}^{n}: \operatorname{supp}(x) \subseteq I\right\},
$$

where $I \subseteq\langle n\rangle$, and $\operatorname{supp}(x)$ is the support of $x$, i.e. the set $\left\{i \in\langle n\rangle: \xi_{i} \neq 0\right\}$ for $x=\left(\xi_{1}, \cdots, \xi_{n}\right)^{T}$. Indeed, as can be readily seen, the mapping $I \longmapsto F_{I}$ is an isomorphism between the lattices $2^{\langle n\rangle}$ and $\mathcal{F}\left(\mathbb{R}_{+}^{n}\right)$. The following result asserts that if $P$ is an $n \times n$ nonnegative matrix, then under this isomorphism, the initial subsets for $P$ correspond to the $P$-invariant faces of $\mathbb{R}_{+}^{n}$.

Theorem 3.1. Let $P$ be an $n \times n$ nonnegative matrix. Denote by $\mathcal{I}$ the lattice of all initial subsets for $P$ and by $\mathcal{F}_{P}$ the lattice of all $P$-invariant faces of $\mathbb{R}_{+}^{n}$. Then the association $I \longmapsto F_{I}$ induces an isomorphism from the lattice $\mathcal{I}$ onto the lattice $\mathcal{F}_{P}$. 
Proof. Let $\triangle: \mathcal{I} \longrightarrow \mathcal{F}_{P}$ be the mapping defined by $\triangle(I)=F_{I}$. First, we need to show that $\triangle$ is a well-defined mapping. Let $I \in \mathcal{I}$. To show that $F_{I}$ is $P$-invariant, take any vector $x \in F_{I}$. Then

$$
\begin{aligned}
\operatorname{supp}(P x) & =\left\{j \in\langle n\rangle: p_{j i} \neq 0 \text { for some } i \in \operatorname{supp}(x)\right\} \\
& \subseteq\{j \in\langle n\rangle: j \text { has access to } \operatorname{supp}(x)\} \\
& \subseteq I,
\end{aligned}
$$

where the last inclusion follows from the fact that $\operatorname{supp}(x) \subseteq I$ and $I$ is an initial subset for $P$. Hence, by definition, we have $P x \in F_{I}$.

Because $\triangle$ is just a restriction of the isomorphism $I \longmapsto F_{I}$ between the lattices $2^{\langle n\rangle}$ and $\mathcal{F}\left(\mathbb{R}_{+}^{n}\right)$, and $\mathcal{I}$ and $\mathcal{F}_{P}$ are respectively sublattices of $2^{\langle n\rangle}$ and $\mathcal{F}\left(\mathbb{R}_{+}^{n}\right), \triangle$ is a one-to-one lattice homomorphism. It remains to show that $\triangle$ is onto.

Let $F$ be a $P$-invariant face. As a face of $\mathbb{R}_{+}^{n}, F$ must be of the form $F_{S}$ for some $S \subseteq\langle n\rangle$; indeed, $S$ is the set $\bigcup_{x \in F} \operatorname{supp}(x)$. Let $i \in S$. Consider any $j \in\langle n\rangle$ which has access to $i$ but is not equal to $i$. Since $j$ has access to $i$, there exists a positive integer $k$ such that the $(j, i)$ entry of $P^{k}$ is nonzero; or, in other words, $j \in \operatorname{supp}\left(P^{k} e_{i}\right)$, where $e_{i}$ is the $i$ th standard unit vector of $\mathbb{R}^{n}$. But $P^{k} e_{i} \in F$, as $e_{i} \in F$ and $F$ is $P$-invariant, so $j \in S$. This shows that $S$ is an initial subset for $P$, and thus establishes the surjectivity of $\triangle$. The proof is complete.

Let $\mathcal{K}$ be a nonempty collection of classes of $P$. A class $\alpha \in \mathcal{K}$ is said to be final in $\mathcal{K}$ if it has no access to other classes of $\mathcal{K}$. If $I$ is a nonempty initial subset for $P$, then as mentioned before, $I$ is the union of all classes in an initial collection $\mathcal{K}$ of classes of $P$. An initial subset $I$ for $P$ is said to be determined by a class $\alpha$ of $P$ if it is the union of all classes of $P$ having access to $\alpha$ (or, in other words, $\alpha$ is the only class final in the initial collection corresponding to $I$ ); in this case, we write $F_{I}$ simply as $F_{\alpha}$. The use of the notation $F_{\alpha}$ here agrees with that of [T-S1, Section 4]. Since we use only small Greek letters to denote classes, the use of this notation should cause no confusion. According to Theorem 3.1, each $F_{\alpha}$ is a $P$-invariant face.

Lemma 3.2. Let $P$ be an $n \times n$ nonnegative matrix. If $\alpha$ is a distinguished class of $P$ associated with the eigenvalue $\lambda$, then $F_{\alpha}$ contains a unique (up to multiples) eigenvector of $P$ corresponding to $\lambda$. Furthermore, this eigenvector belongs to relint $F_{\alpha}$ and is an extremal distinguished eigenvector of $P$.

Proof. First, note that $\left.P\right|_{\operatorname{span} F_{\alpha}}$ can be represented by the principal submatrix of $P$ with rows and columns indexed by the initial subset for $P$ determined by $\alpha$. As such, the spectrum of $\left.P\right|_{\operatorname{span} F_{\alpha}}$ is simply the union of the spectra of all possible submatrices $P_{\beta \beta}$ for which $\beta$ is a class of $P$ that has access to $\alpha$. Since $\alpha$ is a distinguished class, it follows that $\rho\left(\left.P\right|_{\operatorname{span} F_{\alpha}}\right)=\rho\left(P_{\alpha \alpha}\right)=\lambda$. According to the Perron-Frobenius theorem, there exists an eigenvector $x$ of $P$ corresponding to $\lambda$ that lies in $F_{\alpha}$. If $x \in \operatorname{rbd} F_{\alpha}$, then $\Phi(x)$ is a $P$-invariant face properly included in $F_{\alpha}$, and by Theorem 3.1 there is an initial subset $I$ for $P$ properly included in the initial subset determined by $\alpha$ such that $\Phi(x)=F_{I}$. But then $\rho_{\Phi(x)}=\rho\left(P_{I I}\right)<\lambda$, which contradicts our assumption on $x$. This shows that $x \in \operatorname{relint} F_{\alpha}$.

If $P$ has an eigenvector in $F_{\alpha}$ corresponding to $\lambda$, which is not a multiple of $x$, then we readily obtain an eigenvector corresponding to $\lambda$ that lies in $\operatorname{rbd} F_{\alpha}$. But as we have just shown, this would lead to a contradiction. This proves the uniqueness 
of the desired eigenvector $x$. It also follows that $x$ is an extremal distinguished eigenvector.

We now offer a cone-theoretic proof of the following Frobenius -Victory theorem, first named in [T-S1] (see Theorem 2.1 and the subsequent remarks there).

Theorem 3.3. Let $P$ be an $n \times n$ nonnegative matrix.

(i) A real number $\lambda$ is a distinguished eigenvalue of $P$ (for $\mathbb{R}_{+}^{n}$ ) if and only if there exists a distinguished class $\alpha$ of $P$ such that $\rho\left(P_{\alpha \alpha}\right)=\lambda$.

(ii) If $\alpha$ is a distinguished class of $P$, then there is a unique (up to multiples) nonnegative eigenvector $x^{\alpha}=\left(\xi_{1}, \cdots, \xi_{n}\right)^{T}$ corresponding to $\rho\left(P_{\alpha \alpha}\right)$ with the property that $\xi_{i}>0$ if and only if $i$ has access to $\alpha$.

(iii) For each distinguished eigenvalue $\lambda$ of $P$, the cone $\mathcal{N}(\lambda I-P) \cap \mathbb{R}_{+}^{n}$ is simplicial and its extreme vectors are precisely all the distinguished eigenvectors of $P$ of the form $x^{\alpha}$ as given in (ii), where $\alpha$ is a distinguished class such that $\rho\left(P_{\alpha \alpha}\right)=\lambda$.

Proof. (i) The " if " part follows from Lemma 3.2. To prove the " only if " part, choose a distinguished $A$-invariant face $F$ of $\mathbb{R}_{+}^{n}$ associated with $\lambda$. By Theorem $3.1, F=F_{I}$ for some nonempty initial subset $I$ for $P$. Note that there can be only one class final in the initial collection of classes corresponding to $I$, say $\alpha$ (i.e. $F_{I}=F_{\alpha}$ ), and also that $\alpha$ is a distinguished class associated with $\lambda$; otherwise, it would be possible to choose a nonempty initial subset $I^{\prime}$ properly included in $I$, and hence $F_{I^{\prime}}$ properly included in $F_{I}$, such that $\rho_{F_{I^{\prime}}}=\rho_{F_{I}}=\lambda$, contradicting the assumption that $F$ is a distinguished $A$-invariant face.

(ii) follows from Lemma 3.2 (and the definition of $F_{\alpha}$ ).

(iii) By Lemma 3.2 each distinguished eigenvector of the form $x^{\alpha}$, where $\alpha$ is a distinguished class associated with $\lambda$, is an extreme vector of $\mathcal{N}(\lambda I-P) \cap \mathbb{R}_{+}^{n}$. Conversely, if $x$ is a (nonzero) extreme vector of $\mathcal{N}(\lambda I-P) \cap \mathbb{R}_{+}^{n}$, then, as can be readily shown, $\Phi(x)$ is a distinguished $A$-invariant face of $\mathbb{R}_{+}^{n}$. From the proof of the " only if " part of (i), then we have $\Phi(x)=F_{\alpha}$ for some distinguished class $\alpha$ associated with $\lambda$. But by Lemma 3.2 the eigenvector of $P$ corresponding to $\lambda$ that lies in $F_{\alpha}$ is unique, so $x=x^{\alpha}$. This proves that the extreme vectors of the cone $\mathcal{N}(\lambda I-P) \cap \mathbb{R}_{+}^{n}$ are precisely those of the given form. That the cone $\mathcal{N}(\lambda I-P) \cap \mathbb{R}_{+}^{n}$ is simplicial, i.e. its set of distinct (up to multiples) extreme vectors is linearly independent, follows from the combinatorial properties of the supports of these vectors.

Using the Frobenius-Victory theorem, in Tam3 Theorem 5.2] a cone-theoretic proof is offered for the well-known nonnegative basis theorem for a nonnegative matrix (or a singular $M$-matrix). [A stronger result, known as the preferred basis theorem, has also been found. See [H-S].] Hershkowitz and Schneider $[\mathrm{H}-\mathrm{S}$, Section 5] have also extended the nonnegative basis theorem to cover the case of a distinguished eigenvalue. We reformulate their result as Theorem 3.4 below, and offer an independent proof which is based on the nonnegative basis theorem and makes use of cone-theoretic arguments.

Theorem 3.4. Let $P$ be an $n \times n$ nonnegative matrix, and let $\lambda$ be a distinguished eigenvalue of $P$. Let $\alpha_{1}, \cdots, \alpha_{p}$ be all the semi-distinguished classes of $P$ associated with $\lambda$. Then the generalized eigenspace of $P$ corresponding to $\lambda$ contains nonnegative vectors $x^{\alpha_{1}}, \cdots, x^{\alpha_{p}}$ such that $x^{\alpha_{i}} \in \operatorname{relint} F_{\alpha_{i}}$ for each $i$. Furthermore, any 
such collection forms a basis for the subspace spanned by the nonnegative generalized eigenvectors of $P$ corresponding to $\lambda$.

Before we come to the proof of Theorem 3.4, we need the following lemma.

Lemma 3.5. Let $P$ be an $n \times n$ nonnegative matrix. If $F$ is a $P$-invariant face of $\mathbb{R}_{+}^{n}$ which contains in its relative interior a generalized eigenvector of $P$, then $F$ must be of the form $F_{I}$, where $I$ is a nonempty initial subset for $P$ with the property that each class final in the initial collection of classes corresponding to $I$ is a semi-distinguished class associated with $\rho_{F}$.

Proof. By Theorem 3.1, $F$ is of the form $F_{I}$ for some initial subset $I$ for $P$. Denote by $B$ the principal submatrix of $P$ with columns and rows indexed by $I$. Since $P$ has a generalized eigenvector that lies in relint $F$, the nonnegative matrix $B$ has a positive generalized eigenvector. Hence, by [Tam3, Theorem 5.1] $\rho_{F}(=\rho(B))$ is the only distinguished eigenvalue of $B^{T}$ (for the corresponding nonnegative orthant). By the Frobenius-Victory theorem it follows that every initial class of $B^{T}$, and hence every final class of $B$, is associated with $\rho_{F}$. But the final classes of $B$ are precisely the classes final in the initial collection of classes corresponding to $I$, hence our assertion follows.

Proof of Theorem 3.4. Let $\alpha$ be any semi-distinguished class of $P$ associated with $\lambda$. Let $Q$ be the principal submatrix of $P$ with rows and columns indexed by the initial subset determined by $\alpha$. Then

$$
\rho(Q)=\max \left\{\rho\left(P_{\beta \beta}\right): \beta \text { has access to } \alpha\right\}=\lambda,
$$

where the last equality follows from the assumption that $\alpha$ is a semi-distinguished class of $P$. An application of the first part of the nonnegative basis theorem (see Tam3, Theorem 5.2]) to $Q$ guarantees that there exists a positive generalized eigenvector $w$ of $Q$ corresponding to $\lambda$. For convenience, we index the components of $w$ by the initial subset of $P$ determined by $\alpha$. Let $x^{\alpha}$ be the vector in $\mathbb{R}_{+}^{n}$ defined by saying that $\left(x^{\alpha}\right)_{i}$ equals $w_{i}$ if $i$ has access to $\alpha$, and equals zero otherwise. Then $x^{\alpha}$ is the desired generalized eigenvector of $P$ corresponding to $\lambda$ which is associated with the semi-distinguished class $\alpha$.

It is clear that any collection of vectors $x^{\alpha}$, with one vector for each semidistinguished class $\alpha$ associated with $\lambda$, forms a linearly independent family, in view of the combinatorial properties of these vectors. To complete the proof, it remains to show that if $u$ is a nonnegative generalized eigenvector of $P$ corresponding to $\lambda$, then $u$ is spanned by any such collection of vectors $x^{\alpha}$. For that purpose, it suffices to show that $\operatorname{supp}(u) \subseteq I$, where we use $I$ to denote the union of all classes of $P$ having access to at least one semi-distinguished class associated with $\lambda$. This is because we can then apply the nonnegative basis theorem to the principal submatrix of $P$ with rows and columns indexed by $I$ to draw the desired conclusion.

Let $u$ be any nonnegative generalized eigenvector of $P$ corresponding to $\lambda$. By Lemma 2.1 the face $\Phi\left((I+P)^{n-1} u\right)$ is the smallest $P$-invariant face of $\mathbb{R}_{+}^{n}$ containing $u$, and by Theorem 3.1 it must be of the form $F_{S}$, for a unique initial subset $S$ for $P$. (In fact, $S$ is the smallest initial subset for $P$ including $\operatorname{supp}(u)$, i.e. it is the union of all classes of $P$ having access to $\operatorname{supp}(u)$.) Since $F_{S}$ contains a generalized eigenvector of $P$ corresponding to $\lambda$ in its relative interior, namely, the vector $(I+P)^{n-1} u$, by Lemma 3.5 each class final in the initial collection of classes 
corresponding to $S$ is a semi-distinguished class associated with $\lambda$. Hence, we have

$$
\operatorname{supp}(u) \subseteq \operatorname{supp}\left((I+P)^{n-1} u\right)=S \subseteq I,
$$

as desired.

The following characterizations of different types of $P$-invariant faces associated with a nonnegative matrix $P$ motivate much of our subsequent work in this paper.

Theorem 3.6. Let $P$ be an $n \times n$ nonnegative matrix. Let $I$ be an initial subset for P. Then:

(i) $F_{I}$ is a minimal nonzero $P$-invariant face if and only if $I$ is an initial class of $P$.

(ii) $F_{I}$ is a nonzero $P$-invariant join-irreducible face if and only if $I$ is an initial subset determined by a single class.

(iii) $F_{I}$ is a $P$-invariant face which contains in its relative interior a generalized eigenvector (respectively, an eigenvector) of $P$ corresponding to $\lambda$ if and only if $I$ is a nonempty initial subset such that each class final in the initial collection of classes corresponding to $I$ is a semi-distinguished (respectively, distinguished) class associated with $\lambda$.

(iv) $F_{I}$ is a $P$-invariant join-irreducible face which contains in its relative interior a generalized eigenvector (respectively, an eigenvector) of $P$ corresponding to $\lambda$ if and only if $I$ is an initial subset determined by a semi-distinguished (respectively, distinguished) class associated with $\lambda$.

Proof. As in the proof of Theorem 3.1, we denote by $\triangle$ the isomorphism from $\mathcal{I}$, the lattice of all initial subsets for $P$, onto $\mathcal{F}_{P}$, the lattice of all $P$-invariant faces of $\mathbb{R}_{+}^{n}$, given by $\triangle(I)=F_{I}$.

(i): The minimal nonempty members of $\mathcal{I}$ are precisely those initial subsets of $P$ determined by an initial class. Under the lattice isomorphism $\triangle$, they clearly correspond to the minimal nonzero $P$-invariant faces of $\mathbb{R}_{+}^{n}$.

(ii): Note that the nonempty join-irreducible members of the lattice $\mathcal{I}$ are precisely those initial subsets determined by a single class, whereas the nonzero joinirreducible members of $\mathcal{F}_{P}$ are the nonzero $P$-invariant join-irreducible faces. But $\triangle$ sends the empty set to the zero face and as a lattice isomorphism it preserves the join-irreducibility property, so our assertion follows.

(iii): "Only if " part: The " generalized eigenvector" case follows immediately from Lemma 3.5. To prove the "eigenvector" case, we denote by $B$, as in the proof of Lemma 3.5, the principal submatrix of $P$ with columns and rows indexed by $I$. Here, instead of using [Tam3, Theorem 5.1], we apply Tam3, Theorem 5.4] to $B$ and deduce that $\nu_{\rho_{F}}(B)=1$, and also that $I$ is a nonempty initial subset with the property that each class final in the initial collection of classes corresponding to $I$ is a semi-distinguished class (of $B$, or equivalently, of $P$ ) associated with $\rho_{F}$. It remains to show that each class final in the initial collection corresponding to $I$ is, in fact, a distinguished class. (Here, if we apply the Rothblum index theorem, we can already draw the desired conclusion. But, providing an alternative conetheoretic proof for the Rothblum index theorem and its extension is one of the purposes of this paper, so we proceed instead as follows.) Suppose that there is a class, say $\beta$, which is final in the initial collection corresponding to $I$ but is not a distinguished class. Then by Theorem 3.4 we can find a nonnegative generalized eigenvector $x=\left(\xi_{i}\right)^{T}$ of $B$ corresponding to $\rho_{F}$ with the property that $\xi_{i}>0$ if 
and only if $i$ has access to $\beta$. From a consideration of supports of vectors, it is clear that $x$ cannot be written as a linear combination of vectors of the form $x^{\alpha}$ as given in Theorem 3.3(ii), where $\alpha$ is a distinguished class of $B$ associated with $\rho_{F}$. Hence, it follows that $x$ is a generalized eigenvector of order greater than 1 . Thus, we obtain $\nu_{\rho_{F}}(B) \geq 2$, which is a contradiction.

"If " part: We give the proof for the "generalized eigenvector " case, and omit the proof for the "eigenvector" case, which depends on the use of the Frobenius Victory theorem and is similar and easier. We denote by $\mathcal{K}$ the initial collection of classes of $P$ corresponding to the initial subset $I$.

Let $\alpha_{1}, \cdots, \alpha_{k}$ be the classes final in $\mathcal{K}$. By our assumption on $I$, each $\alpha_{i}$ is a semi-distinguished class associated with the eigenvalue $\lambda$. By Theorem 3.4, for each $i, 1 \leq i \leq k$, there is a generalized eigenvector, say $x_{i}$, of $P$ corresponding to $\lambda$ that lies in relint $F_{\alpha_{i}}$. Since $I$ is an initial subset, it follows that the vector $\sum_{i=1}^{k} x_{i}$ is a generalized eigenvector of $P$ corresponding to $\lambda$ that lies in relint $F_{I}$.

(iv): The "only if " part and the "if " part follow respectively from the corresponding parts of (ii) and (iii).

\section{The SPECTRAL PAIR OF A FACE}

Let $A \in \pi(K)$. To any face $F$ of $K, A$-invariant or not, we are going to assign an ordered pair of nonnegative real numbers, known as the spectral pair of $F$ relative to $A$. Such spectral pairs of faces will turn out to be useful in our later investigations. But first we need the concept of a spectral pair of a vector relative to an arbitrary (square) matrix, and for this purpose we introduce the concept of the order of a vector relative to a matrix. We thus extend the usual concept of the order of a generalized eigenvector.

Let $A \in \mathcal{M}_{n}$ and let $x$ be a nonzero vector of $\mathbb{C}^{n}$. Consider the (unique) representation of $x$ as a sum of generalized eigenvectors of $A$, say,

$$
x=x_{1}+\cdots+x_{m},
$$

where $x_{1}, \cdots, x_{m}$ are generalized eigenvector of $A$ corresponding respectively to distinct eigenvalues $\lambda_{1}, \cdots, \lambda_{m}$. By definition, $\rho_{x}(A)$ equals $\max _{1 \leq i \leq m}\left|\lambda_{i}\right|$. By the order of $x$ relative to $A$, denoted by $\operatorname{ord}_{A}(x)$, we mean the maximum of the orders of generalized eigenvectors, each corresponding to an eigenvalue of modulus $\rho_{x}(A)$, that appear in the above representation of $x$. Now we denote the ordered pair $\left(\rho_{x}(A), \operatorname{ord}_{A}(x)\right)$ by $\operatorname{sp}_{A}(x)$ and refer to it as the spectral pair of $x$ relative to $A$. We also set $\operatorname{sp}_{A}(\mathbf{0})=(0,0)$ to take care of the zero vector $\mathbf{0}$. We denote by $\preceq$ the lexicographic ordering between ordered pairs of real numbers given by

$$
\left(\xi_{1}, \xi_{2}\right) \preceq\left(\eta_{1}, \eta_{2}\right) \text { if either } \xi_{1}<\eta_{1} \text {, or } \xi_{1}=\eta_{1} \text { and } \xi_{2} \leq \eta_{2} \text {. }
$$

We also write $\left(\xi_{1}, \xi_{2}\right) \prec\left(\eta_{1}, \eta_{2}\right)$, if $\left(\xi_{1}, \xi_{2}\right) \preceq\left(\eta_{1}, \eta_{2}\right)$ but the equality does not hold in the usual sense.

Using the definition of the spectral pair of a vector (relative to a matrix), it is easy to establish the following:

Remark 4.1. For any $A \in \mathcal{M}_{n}, x, y \in \mathbb{C}^{n}, 0 \neq \lambda \in \mathbb{C}$, we have:

(i) $\operatorname{sp}_{A}(\lambda x)=\operatorname{sp}_{A}(x)$.

(ii) $\operatorname{sp}_{A}(A x)$ equals $\operatorname{sp}_{A}(x)$ if $\rho_{x}(A)>0$, and equals $\left(0, \operatorname{ord}_{A}(x)-1\right)$ if $\rho_{x}(A)=0$ and $x \neq \mathbf{0}$.

(iii) $\operatorname{sp}_{A}(x+y) \preceq \max \left\{\operatorname{sp}_{A}(x), \operatorname{sp}_{A}(y)\right\}$, where the maximum is taken in the sense of lexicographic ordering. 
Part (iii) of Remark 4.1 is a strengthening of the known result [T-W, Lemma 2.3 that if $x_{1}, x_{2} \in \mathbb{C}^{n}$, then

$$
\rho_{x_{1}+x_{2}}(A) \leq \max \left\{\rho_{x_{1}}(A), \rho_{x_{2}}(A)\right\}
$$

Indeed, we have indicated an easy alternative proof.

Here we would like to mention some relevant results which we need later. For any $n \times n$ complex matrix $A$ and any eigenvalue $\lambda$ of $A$, let $E_{\lambda}^{(0)}$ denote the projection of $\mathbb{C}^{n}$ onto the generalized eigenspace $\mathcal{N}\left((\lambda I-A)^{n}\right)$ along the direct sum of other generalized eigenspaces of $A$. Also define the components of $A$ by

$$
E_{\lambda}^{(k)}=(A-\lambda I)^{k} E_{\lambda}^{(0)}, \quad k=0,1, \cdots .
$$

In [Schn2 Theorem 5.2 (iii)] it is proved that if $K$ is a proper cone and $A \in \pi(K)$, then $\left.E_{\rho}^{(\nu-1)}\right|_{\mathbb{R}^{n}} \in \pi(K)$, where $\rho=\rho(A)$ and $\nu=\nu_{\rho(A)}(A)$. Furthermore, we have $\operatorname{rank} E_{\rho}^{(\nu-1)}=\mu_{\rho}(A)$, where $\mu_{\rho}(A)$ is the exponent of $\rho(A)$, i.e. the number of Jordan blocks of $A$ of maximal order corresponding to $\rho(A)$. (This latter fact is always true; it does not depend on the condition that $A \in \pi(K)$.) Thus, $E_{\rho}^{(\nu-1)} K$ is a cone of dimension $\mu_{\rho}(A)$ which consists of some of the distinguished eigenvectors of $A$ corresponding to $\rho(A)$, together with the zero vector. For the purpose of later references, we make a note of the following.

Remark 4.2. If $A \in \pi(K)$, then there exist $\mu_{\rho}(A)$ (the exponent of $\rho(A)$ ) linearly independent distinguished eigenvectors of $A$ corresponding to $\rho(A)$, such that each is associated with a maximal Jordan block, in the sense that each is a vector of the form $(A-\rho(A) I)^{\nu-1} z$ for some generalized eigenvector $z$ of order $\nu=\nu_{\rho(A)}(A)$ corresponding to $\rho(A)$.

Now we make an important observation.

Lemma 4.3. Let $A \in \pi(K)$. For any $x \in \operatorname{int} K$, we have $\operatorname{sp}_{A}(x)=\left(\rho(A), \nu_{\rho(A)}(A)\right)$. Proof. As we have mentioned above, $\left.E_{\rho}^{(\nu-1)}\right|_{\mathbb{R}^{n}}$ is nonzero and belongs to $\pi(K)$, where $\nu=\nu_{\rho(A)}(A)$. For any $x \in \operatorname{int} K$, necessarily $E_{\rho}^{(\nu-1)} x \neq \mathbf{0}$; otherwise, $\left.E_{\rho}^{(\nu-1)}\right|_{\mathbb{R}^{n}}$, and hence $E_{\rho}^{(\nu-1)}$, is the zero operator. Hence, by definition of $E_{\rho}^{(\nu-1)}$, in the representation of $x$ as a sum of generalized eigenvectors of $A$, there must be a term which is a generalized eigenvector of $A$ corresponding to $\rho(A)$, and furthermore the order of this generalized eigenvector must be $\nu$. Moreover, by the Perron-Schaefer condition for $A, \nu$ is equal to the maximum of the orders of generalized eigenvectors, each corresponding to an eigenvalue belonging to the peripheral spectrum of $A$, that appear in the representation of $x$. Therefore, we have

$$
\operatorname{sp}_{A}(x)=(\rho(A), \nu) .
$$

An application of Lemma 4.3 to the restriction of $A(\in \pi(K))$ to the linear span of an $A$-invariant face $F$ immediately yields

Theorem 4.4. Let $A \in \pi(K)$, and let $F$ be an $A$-invariant face of $K$. Then for any vector $x \in$ relint $F$, we have $\operatorname{sp}_{A}(x)=\left(\rho_{F}, \nu_{\rho_{F}}\left(\left.A\right|_{\operatorname{span} F}\right)\right)$.

By Theorem 4.4 and the definition of the spectral pair of a vector, we have the following. 
Corollary 4.5. Let $A \in \pi(K)$. If $F$ is an $A$-invariant face of $K$, then $\rho_{x}(A)=\rho_{F}$ for any $x \in \operatorname{relint} F$.

Theorem 4.4 also says that, for any $A$-invariant face $F$, the value of the spectral pair $\operatorname{sp}_{A}(x)$ is independent of the choice of $x$ from relint $F$. Now we show that the same is also true for any face $F$ of $K$.

Theorem 4.6. Let $A \in \pi(K)$, and let $F$ be a face of $K$. For any $x \in \operatorname{relint} F$, we have

$$
\operatorname{sp}_{A}(x)=\left(\rho_{\widehat{F}}, \nu_{\widehat{F}}\left(\left.A\right|_{\text {span } \widehat{F}}\right)\right),
$$

where $\widehat{F}$ is the smallest $A$-invariant face of $K$ including $F$.

Proof. Choose any positive real number $\alpha$ for which $-\alpha$ is not an eigenvalue of $A$. Consider any vector $x$ chosen from relint $F$. For convenience, denote the vector $(\alpha I+A)^{n-1} x$ by $\widehat{x}$. Then the proof of Lemma 2.1 also shows that $\Phi(\widehat{x})$ equals $\widehat{F}$. Let

$$
x=x_{1}+\cdots+x_{m}
$$

where $x_{1}, \cdots, x_{m}$ are generalized eigenvectors of $A$ corresponding to distinct eigenvalues $\lambda_{1}, \cdots, \lambda_{m}$ respectively. Applying $(\alpha I+A)^{n-1}$ to both sides of (1), we obtain

$$
\widehat{x}=(\alpha I+A)^{n-1} x_{1}+\cdots+(\alpha I+A)^{n-1} x_{m} .
$$

By our choice of $\alpha,(\alpha I+A)^{n-1}$ is nonsingular; hence, each $(\alpha I+A)^{n-1} x_{i}$ is a generalized eigenvector of $A$ corresponding to $\lambda_{i}$, and is of the same order as $x_{i}$. From the definition of the spectral pair of a vector, it is now clear that we have

$$
\operatorname{sp}_{A}(x)=\operatorname{sp}_{A}(\widehat{x})=\left(\rho_{\widehat{F}}, \nu_{\widehat{F}}\left(\left.A\right|_{\text {span } \widehat{F}}\right)\right),
$$

where the last equality follows from Theorem 4.4.

By Theorem 4.6, for any face $F$ of $K$, the value of the spectral pair $\operatorname{sp}_{A}(x)$ is independent of the choice of $x$ from relint $F$. Henceforth, we shall denote this constant value by $\operatorname{sp}_{A}(F)$, and refer to it as the spectral pair of $F$ relative to $A$. The spectral pair of a face will prove to be a useful concept, though it is not an extension of an existing concept for nonnegative matrices. This probably is due to the fact that its definition implicitly involves the Perron-Schaefer condition, a characterizing property of a cone-preserving map.

In passing, we also take note of the following by-products.

Theorem 4.7. Let $A \in \pi(K)$. If $\mathbf{0} \neq x \in K$, then there is always a generalized eigenvector $y$ of $A$ corresponding to $\rho_{x}(A)$ that appears as a term in the representation of $x$ as a sum of generalized eigenvectors of $A$. Furthermore, $\operatorname{ord}_{A}(x)=$ $\operatorname{ord}_{A}(y)$.

Proof. If the face $\Phi(x)$ is $A$-invariant, our assertion follows from the proof of Lemma 4.3. In view of the proof of Theorem 4.6, our assertion also holds for the case when $\Phi(x)$ is not $A$-invariant.

The following result should also be clear.

Corollary 4.8. Let $A \in \pi(K)$, and let $\mathbf{0} \neq x \in K$. Denote $\operatorname{ord}_{A}(x)$ by $m$. Then $\left(A-\rho_{x}(A) I\right)^{m-1} E_{\rho_{x}(A)}^{(0)} x$ is a distinguished eigenvector of $A$ corresponding to $\rho_{x}(A)$. 
In the next result we collect together fundamental properties of spectral pairs of faces (or, of vectors in the cone $K$ ).

Theorem 4.9. Let $A \in \pi(K)$.

(i) For any faces $F, G$ of $K$, we have:

(a) $\operatorname{sp}_{A}(F)=\operatorname{sp}_{A}(\widehat{F})$, where $\widehat{F}$ is the smallest A-invariant face of $K$ including $F$.

(b) If $F \subseteq G$, then $\operatorname{sp}_{A}(F) \preceq \operatorname{sp}_{A}(G)$.

(c) $\operatorname{sp}_{A}(F \vee G)=\max \left\{\operatorname{sp}_{A}(F), \operatorname{sp}_{A}(G)\right\}$, where the maximum is taken in the sense of lexicographic ordering.

(ii) For any vectors $x, y \in K$, we have:

(a) $\operatorname{sp}_{A}(x)=\operatorname{sp}_{A}\left((I+A)^{n-1} x\right)$.

(b) If $x \in \Phi(y)$, then $\operatorname{sp}_{A}(x) \preceq \operatorname{sp}_{A}(y)$.

(c) $\operatorname{sp}_{A}(x+y)=\max \left\{\operatorname{sp}_{A}(x), \operatorname{sp}_{A}(y)\right\}$, where the maximum is taken in the sense of lexicographic ordering.

Proof. As can be readily shown, properties (a), (b) or (c) of part (i) are each equivalent to the corresponding properties of part (ii). So, for each property, (a), (b) or (c), we need only consider either the one in (i) or the one in (ii).

In view of Theorems 4.4 and 4.6 and the definition of the spectral pair of a face, we have $\operatorname{sp}_{A}(F)=\operatorname{sp}_{A}(\widehat{F})$. So we need not consider property (a) further.

Let $F, G$ be faces of $K$ such that $F \subseteq G$. Then clearly we have $\widehat{F} \subseteq \widehat{G}$; thus, $\rho_{\widehat{F}} \leq \rho_{\widehat{G}}$, and if $\rho_{\widehat{F}}=\rho_{\widehat{G}}$ then $\nu_{\widehat{F}}\left(\left.A\right|_{\text {span } \widehat{F}}\right) \leq \nu_{\rho_{\widehat{G}}}\left(\left.A\right|_{\text {span } \widehat{G}}\right)$. That is, we have $\operatorname{sp}_{A}(\widehat{F}) \preceq \operatorname{sp}_{A}(\widehat{G})$, and in view of property (a) of (i), property (b) of (i) follows.

To prove property (c) of (ii), first note that according to Remark 4.1(iii) we always have

$$
\operatorname{sp}_{A}(x+y) \preceq \max \left\{\operatorname{sp}_{A}(x), \operatorname{sp}_{A}(y)\right\} .
$$

Since $x \in \Phi(x+y)$, by property (b) of (ii), $\operatorname{sp}_{A}(x) \preceq \mathrm{sp}_{A}(x+y)$. Similarly, we also have $\operatorname{sp}_{A}(y) \preceq \operatorname{sp}_{A}(x+y)$, and hence

$$
\max \left\{\operatorname{sp}_{A}(x), \operatorname{sp}_{A}(y)\right\} \preceq \operatorname{sp}_{A}(x+y) .
$$

Therefore, the desired equality holds.

An immediate consequence of property (c) of Theorem 4.9 (i) is that, if $F$ is an $A$-invariant face that satisfies $\operatorname{sp}_{A}(F) \succ \operatorname{sp}_{A}(G)$ for all $A$-invariant faces $G$ properly included in $F$, then $F$ is $A$-invariant join-irreducible. The converse of this result is not true. For instance, take $A$ to be the nonnegative matrix: $\left(\begin{array}{ll}1 & 1 \\ 0 & 0\end{array}\right)$. Then $\mathbb{R}_{+}^{2}$ and $\Phi\left(e_{1}\right)$ are the only nonzero $A$-invariant faces of $\mathbb{R}_{+}^{2}$, so that $\mathbb{R}_{+}^{2}$ is an $A$-invariant join-irreducible face of itself. However, we have $\operatorname{sp}_{A}\left(\mathbb{R}_{+}^{2}\right)=(1,1)=\operatorname{sp}_{A}\left(\Phi\left(e_{1}\right)\right)$.

By Remark 4.1(i), (ii) and properties (b), (c) of Theorem 4.9(ii), we also readily obtain the following.

Corollary 4.10. Let $A \in \pi(K)$. For any nonnegative real number $\lambda$ and any positive integer $k$, the set

$$
F_{\lambda, k}=\left\{x \in K: \operatorname{sp}_{A}(x) \preceq(\lambda, k)\right\}
$$

is an A-invariant face of $K$. Furthermore, for any nonnegative real numbers $\lambda_{1}, \lambda_{2}$, and positive integers $k_{1}, k_{2}$, if $\left(\lambda_{1}, k_{1}\right) \preceq\left(\lambda_{2}, k_{2}\right)$, then $F_{\lambda_{1}, k_{1}} \subseteq F_{\lambda_{2}, k_{2}}$. 
For a similar reason, for any positive real number $\lambda$, the set

$$
F_{\lambda}=\left\{x \in K: \rho_{x}(A)<\lambda\right\}
$$

is an $A$-invariant face of $K$; the greater $\lambda$ is, the larger is the face $F_{\lambda}$. In the case of a positive linear operator $T$ defined on a real Banach lattice $E$, Meyer-Nieberg (see MN1 or [MN2, pp. 293]) has discovered a class of $T$-invariant ideals $J_{\lambda}$, which is precisely the linear span of our $F_{\lambda}$ when the underlying Banach lattice is $\mathbb{R}^{n}$ (with positive cone $\mathbb{R}_{+}^{n}$ ). We explain.

For each real number $\lambda>0$, the ideal $J_{\lambda}$ introduced by Meyer-Nieberg is given by

$$
J_{\lambda}=\left\{x \in E: \text { the series } \sum_{j=0}^{\infty} T^{j}|x| / \lambda^{j+1} \text { is convergent }\right\} .
$$

Here we follow the standard notation of Banach lattice theory (for reference, see Scha] ) and use $|x|$ to denote the modulus of $x$. First, we show that

$$
F_{\lambda}=\left\{x \in \mathbb{R}_{+}^{n}: \text { the series } \sum_{j=0}^{\infty} T^{j} x / \lambda^{j+1} \text { is convergent }\right\} .
$$

If $x \in F_{\lambda}$, then by definition, $\rho_{x}(T)<\lambda$. But $\rho_{x}(T)=\rho\left(\left.T\right|_{W_{x}}\right)$ (see $\mathrm{T}-\mathrm{W}$, Theorem 2.2]), where $W_{x}$ is the cyclic space generated by $x$, so $\left(\lambda I-\left.T\right|_{W_{x}}\right)^{-1}$ exists and is given by

$$
\left(\lambda I-\left.T\right|_{W_{x}}\right)^{-1}=\lambda^{-1} \sum_{j=0}^{\infty}\left(\left.T\right|_{W_{x}} / \lambda\right)^{j} .
$$

Hence, the series $\sum_{j=0}^{\infty} T^{j} x / \lambda^{j+1}=\sum_{j=0}^{\infty}\left(\left.T\right|_{W_{x}}\right)^{j} x / \lambda^{j+1}$ is convergent. Conversely, if the series $\sum_{j=0}^{\infty} T^{j} x / \lambda^{j+1}$ converges, then

$$
\lim _{m \rightarrow \infty}(T / \lambda)^{m}\left(T^{i} x\right)=\mathbf{0} \text { for } i=0,1,2, \cdots,
$$

and hence

$$
\lim _{m \rightarrow \infty}(T / \lambda)^{m} y=\mathbf{0} \text { for every } y \in W_{x} .
$$

It follows that $\lim _{m \rightarrow \infty}\left(\left.T\right|_{W_{x}} / \lambda\right)^{m}=\mathbf{0}$, and so $\rho_{x}(T)<\lambda$. This establishes our claim on the set $F_{\lambda}$.

For any $x \in \mathbb{R}^{n}$, denote the positive part and the negative part of $x$ respectively by $x^{+}$and $x^{-}$. Let $x \in \operatorname{span} F_{\lambda}$. Since $F_{\lambda}$ is a face of $\mathbb{R}_{+}^{n}$, it is clear that $x^{+}$and $x^{-}$both belong to $F_{\lambda}$. Hence, both of the series $\sum_{j=0}^{\infty} T^{j} x^{+} / \lambda^{j+1}$ and $\sum_{j=0}^{\infty} T^{j} x^{-} / \lambda^{j+1}$ converge, and so does the series $\sum_{j=0}^{\infty} T^{j}|x| / \lambda^{j+1}$, as $|x|=$ $x^{+}+x^{-}$. This proves the inclusion span $F_{\lambda} \subseteq J_{\lambda}$. Conversely, if $x \in J_{\lambda}$, then it is clear that both of the series $\sum_{j=0}^{\infty} T^{j} x^{+} / \lambda^{j+1}$ and $\sum_{j=0}^{\infty} T^{j} x^{-} / \lambda^{j+1}$ converge; hence, $x^{+}, x^{-} \in F_{\lambda}$, and so $x=x^{+}-x^{-} \in \operatorname{span} F_{\lambda}$. This proves the reverse inclusion and hence the desired equality.

So, in a sense, our Corollary 4.10 is an extension and also a refinement of the above-mentioned result of Meyer-Nieberg for the finite-dimensional case.

Each property about spectral pairs given in Theorem 4.9 clearly implies a corresponding property about (local) spectral radii. For instance, by property (c) of (i) we have the following: If $F, G$ are $A$-invariant faces of $K$, then $\rho_{F \vee G}=$ $\max \left\{\rho_{F}, \rho_{G}\right\}$. We leave it to the reader to formulate the details. 
Now we give equivalent conditions for an $A$-invariant face of $K$ to be distinguished.

Theorem 4.11. Let $A \in \pi(K)$, and let $F$ be a face of $K$. The following conditions are equivalent:

(a) $F$ is a distinguished A-invariant face of $K$.

(b) $F$ is an A-invariant face, there is a unique (up to positive multiples) eigenvector of $A$ in $F$ that corresponds to $\rho_{F}$, and this eigenvector lies in relint $F$.

(c) $F=\Phi(x)$ for some extremal distinguished eigenvector of $A$.

(d) $F$ is an A-invariant join-irreducible face of $K$, which contains in its relative interior an eigenvector of $A$.

Proof. (a) $\Longrightarrow$ (b). Let $x$ be an eigenvector of $A$ corresponding to $\rho_{F}$ that lies in $F$. If $x \notin \operatorname{relint} F$, then $\Phi(x)$ is a nonzero $A$-invariant face properly included in $F$ such that $\rho_{\Phi(x)}=\rho_{F}$. This contradicts the assumption that $F$ is a distinguished $A$-invariant face. If $x \in \operatorname{relint} F$ and there is an eigenvector, not a multiple of $x$, corresponding to $\rho_{F}$ that lies in $F$, then we readily obtain an eigenvector corresponding to $\rho_{F}$ that lies in $\operatorname{rbd} F$, and by the first part of our argument we also obtain a contradiction.

The equivalence of (b) and (c) is clear.

(b) $\Longrightarrow$ (a). Suppose that there is a nonzero $A$-invariant face $G$ properly included in $F$ such that $\rho_{G}=\rho_{F}$. Then there exists an eigenvector of $A$ corresponding to $\rho_{F}$ that lies in $\operatorname{rbd} F$. This contradicts (b).

(a) $\Longrightarrow(\mathrm{d})$. By condition (b), which is already proved to be equivalent to condition (a), $A$ has an eigenvector that lies in relint $F$. Let $F_{1}, F_{2}$ be $A$-invariant faces such that $F=F_{1} \vee F_{2}$. Then $\rho_{F}=\max \left\{\rho_{F_{1}}, \rho_{F_{2}}\right\}=\rho_{F_{1}}$, say. Since $F$ is distinguished $A$-invariant, it follows that $F_{1}=F$. This proves the $A$-invariant join-irreducibility of $F$.

(d) $\Longrightarrow$ (c). By condition (d) $A$ has an eigenvector $x$ that lies in relint $F$ (and corresponds to $\rho_{F}$ ). Assume to the contrary that $x$ is not an extremal distinguished eigenvector. Then there exist eigenvectors $x_{1}, x_{2}$ of $A$ corresponding to $\rho_{F}$ such that both vectors belong to $\operatorname{rbd} F$ and $x_{1}+x_{2}=x$. Then $\Phi\left(x_{1}\right)$ and $\Phi\left(x_{2}\right)$ are both $A$ invariant faces of $K$ properly included in $F$ such that $\Phi\left(x_{1}\right) \vee \Phi\left(x_{2}\right)=\Phi\left(x_{1}+x_{2}\right)=$ $F$. This contradicts the assumption that $F$ is $A$-invariant join-irreducible.

By Theorem 3.6(iv), if $P$ is a nonnegative matrix and $I$ is an initial subset for $P$, then $I$ is determined by a semi-distinguished (respectively, distinguished) class if and only if $F_{I}$ is a $P$-invariant join-irreducible face which contains in its relative interior a generalized eigenvector (respectively, an eigenvector) of $P$. And in view of the equivalent condition (d) of Theorem 4.11 for a distinguished $A$-invariant face, given any $A \in \pi(K)$, we shall call a face $F$ of $K$ a semi-distinguished $A$-invariant face (associated with $\lambda$ ) if $F$ is an $A$-invariant join-irreducible face which contains in its relative interior a generalized eigenvector of $A$ (corresponding to $\lambda$ ). Clearly a distinguished $A$-invariant face is semi-distinguished $A$-invariant.

Note that we define a nonzero $A$-invariant face $F$ to be distinguished by the property that $\rho_{G}<\rho_{F}$ for any nonzero $A$-invariant face $G$ properly included in $F$. Here a semi-distinguished $A$-invariant face is not defined to be one that satisfies the weaker property obtained by replacing the strict inequality by the weak inequality of the same type, because this latter property is always satisfied! Another reasonable defining property for a semi-distinguished $A$-invariant face is the following: 
$F$ is nonzero $A$-invariant, and $\operatorname{sp}_{A}(G) \prec \operatorname{sp}_{A}(F)$ for any A-invariant face $G$ properly included in $F$.

In view of Theorem 3.6, it is easy to see that in the case when $A$ is a nonnegative matrix, the preceding property is also another equivalent condition for $F$ to be a semi-distinguished $A$-invariant face. Indeed, as we shall see, the same is also true whenever the underlying cone $K$ has the property that the dual cone of each of its faces is facially exposed (which is the case if $K$ is polyhedral), but is not so for a general proper cone $K$.

We shall need the following general result, which is a consequence of the existence of a Jordan basis for a matrix.

Remark 4.12. Let $A \in \mathcal{M}_{n}$ be such that $\rho(A)$ is an eigenvalue. If $z$ is an eigenvector of $A^{T}$ corresponding to $\rho(A)$ which is associated with a maximal Jordan block, then $z$ is orthogonal to every vector $x$ for which $\operatorname{sp}_{A}(x) \prec\left(\rho(A), \nu_{\rho(A)}(A)\right)$.

In the nonnegative matrix case it is easy to determine whether a face of the nonnegative orthant is semi-distinguished invariant or what the spectral pair of a face (or of a nonnegative vector) is, if we apply Theorems 3.1, 3.6, the Rothblum index theorem, and the basic results on spectral pairs of faces (or vectors) obtained in this section. We illustrate this by the following example:

Example 4.13. Consider the following $9 \times 9$ nonnegative matrix:

$$
P=\left[\begin{array}{lllllllll}
1 & 0 & 8 & 0 & 0 & 8 & 0 & 0 & 0 \\
0 & 3 & 6 & 7 & 0 & 0 & 0 & 0 & 0 \\
0 & 0 & 2 & 0 & 1 & 0 & 0 & 0 & 0 \\
0 & 0 & 0 & 3 & 3 & 0 & 4 & 0 & 0 \\
0 & 0 & 0 & 0 & 3 & 5 & 0 & 0 & 0 \\
0 & 0 & 0 & 0 & 0 & 3 & 0 & 0 & 0 \\
0 & 0 & 0 & 0 & 0 & 0 & 0 & 2 & 0 \\
0 & 0 & 0 & 0 & 0 & 0 & 0 & 3 & 3 \\
0 & 0 & 0 & 0 & 0 & 0 & 0 & 0 & 1
\end{array}\right] .
$$

The matrix $P$ has appeared in [Rot]. The reader may refer to [Rot for a diagram of $G(P)$ or draw one of his/her own. Obviously each class of $P$ is a singleton. This is done for simplicity only. As can be readily seen, $I=\{1,2,3,4,5\}$ is an initial subset of $\langle 9\rangle$ determined by the basic class (and hence a semi-distinguished class) $\{5\}$ of $P$. Let $e_{1}, \ldots, e_{9}$ denote the standard unit vectors of $\mathbb{R}^{9}$. By Theorem 3.6(iv), $F_{I}=\Phi\left(e_{1}+e_{2}+e_{3}+e_{4}+e_{5}\right)$ is a semi-distinguished $P$-invariant face. That $F_{I}$ contains in its relative interior a generalized eigenvector (corresponding to $\rho(P)=3$ ) is guaranteed by Theorem 3.4, the nonnegative basis theorem; indeed, the vector $4 e_{1}+e_{2}+e_{3}+e_{4}+e_{5}$ is a generalized eigenvector of $P$ of order three that lies in relint $F_{I}$.

Now we determine the spectral pair of the vector $e_{3}+e_{6}$ relative to $P$. We do it by applying Theorem 4.6. Note that by Theorem 3.1 the smallest $P$-invariant face including $\Phi\left(e_{3}+e_{6}\right)$ is $\Phi\left(e_{1}+\cdots+e_{6}\right)$, as $\langle 6\rangle$ is the smallest initial subset of $\langle 9\rangle$ that includes the subset $\{3,6\}$. Clearly, the principal submatrix $P$ with rows and columns indexed by $\langle 6\rangle$, and hence also the restriction of $P$ to span $\left\{e_{1}, \ldots, e_{6}\right\}$, has spectral radius three and by the Rothblum index theorem its index is four. By Theorem 4.4 and the definition of the spectral pair of a face, the spectral pair of $\Phi\left(e_{1}+\cdots+e_{6}\right)$ is $(3,4)$. Hence, by Theorem 4.6 the spectral pair of the vector $e_{3}+e_{6}$ is also $(3,4)$. A few moments thought should convince the reader that if we 
determine the spectral pair of $e_{3}+e_{6}$ according to the definition, the process would be rather complicated.

\section{An extension of the Rothblum index theorem}

Hershkowitz and Schneider (see [H-S, the paragraph following Theorem 5.10]) proved that the famous Rothblum index theorem (i.e. Theorem A of Section 1) can be extended to cover the case of a distinguished eigenvalue $\lambda$, provided that " basic classes " are replaced by "semi-distinguished classes associated with $\lambda$ " and $\nu$ is replaced by $m_{\lambda}$, the maximal order of distinguished generalized eigenvectors of $P$ corresponding to $\lambda$. This is indeed an extension, because for a nonnegative matrix $P$, as an outcome of the nonnegative basis theorem, we have $m_{\rho(P)}=\nu_{\rho(P)}(P)$.

In the case of a linear mapping preserving a polyhedral cone, we have the following extension of the Rothblum index theorem.

Theorem 5.1. Let $K$ be a polyhedral cone, and let $A \in \pi(K)$. Let $\lambda$ be a distinguished eigenvalue of $A$ for $K$. Denote by $m_{\lambda}$ the maximal order of distinguished generalized eigenvectors of $A$ corresponding to $\lambda$. Then there is a chain of $m_{\lambda}$ distinct semi-distinguished A-invariant faces of $K$ associated with $\lambda$, but there is no such chain with more than $m_{\lambda}$ members.

As can be readily seen, the proof of Theorem 5.1 should consist of two parts: to construct the desired chain of semi-distinguished $A$-invariant faces, and to establish the maximality of its cardinality. The proof of the maximality of cardinality depends on the following result, which has interest of its own.

Lemma 5.2. Let $K$ be a polyhedral cone, and let $A \in \pi(K)$. If $K$ is a semidistinguished $A$-invariant face of itself, then $\operatorname{sp}_{A}(K) \succ \operatorname{sp}_{A}(F)$ for any A-invariant face $F$ properly included in $K$.

Proof. Since int $K$ contains a generalized eigenvector of $A$, by [Tam3 Theorem 5.1] $\rho(A)$ is the only distinguished eigenvalue of $A^{T}$ for $K^{*}$.

We contend that the cone $\mathcal{N}\left(\rho(A) I-A^{T}\right) \cap K^{*}$ is of dimension one. Suppose not. Choose distinct extreme vectors $z_{1}, z_{2}$ from this cone. If $\Phi\left(z_{1}\right) \wedge \Phi\left(z_{2}\right) \neq\{\mathbf{0}\}$, then, being a nonzero $A^{T}$-invariant face, $\Phi\left(z_{1}\right) \wedge \Phi\left(z_{2}\right)$ would contain an eigenvector of $A^{T}$, which necessarily corresponds to $\rho(A)$, and hence is a multiple of $z_{1}$, and also of $z_{2}$, which is a contradiction. Hence, we have $\Phi\left(z_{1}\right) \wedge \Phi\left(z_{2}\right)=\{\mathbf{0}\}$. By our hypothesis, $K^{*}$ is polyhedral. So each face of $K^{*}$ is exposed, and we can write $\Phi\left(z_{i}\right)$ $(i=1,2)$ as $d_{K} \circ d_{K^{*}}\left(\Phi\left(z_{i}\right)\right)$. Thus we have

$$
\begin{aligned}
0 & =\Phi\left(z_{1}\right) \wedge \Phi\left(z_{2}\right) \\
& =d_{K} \circ d_{K^{*}}\left(\Phi\left(z_{1}\right)\right) \wedge d_{K} \circ d_{K^{*}}\left(\Phi\left(z_{2}\right)\right) \\
& =d_{K}\left(d_{K^{*}}\left(\Phi\left(z_{1}\right)\right) \vee d_{K^{*}}\left(\Phi\left(z_{2}\right)\right)\right),
\end{aligned}
$$

which implies that $K$ is the join of the nontrivial $A$-invariant faces $d_{K^{*}}\left(\Phi\left(z_{1}\right)\right)$ and $d_{K^{*}}\left(\Phi\left(z_{2}\right)\right)$. So again we arrive at a contradiction. This establishes our contention. And since $\rho(A)$ is the only distinguished eigenvalue of $A^{T}, A^{T}$ has (up to multiples) a unique distinguished eigenvector.

In view of Remark 4.2, another consequence of our contention is that $A^{T}$ has precisely one maximal Jordan block corresponding to $\rho(A)$. Furthermore, the unique distinguished eigenvector, say $w$, of $A^{T}$ for $K^{*}$ must correspond to this maximal 
block. Thus, we have

$$
(\operatorname{span}\{w\})^{\perp}=\bigoplus_{\lambda \in \sigma(A) \backslash\{\rho(A)\}} \mathcal{N}\left((\lambda I-A)^{n}\right) \oplus U,
$$

where $U$ is the space of generalized eigenvectors of $A$ corresponding to $\rho(A)$ of order less than or equal to $\nu_{\rho(A)}(A)-1$, together with the zero vector.

Now let $F$ be a proper nonzero $A$-invariant face of $K$. Since the $A^{T}$-invariant face $d_{K}(F)$ of $K^{*}$ must contain $w$, we have $\operatorname{span} F \subseteq(\operatorname{span}\{w\})^{\perp}$. In view of the above direct decomposition for $(\operatorname{span}\{w\})^{\perp}$, it follows that if $\operatorname{span} F$ contains a generalized eigenvector of $A$ corresponding to $\rho(A)$, then the order of such a generalized eigenvector must be less than or equal to $\nu_{\rho(A)}(A)-1$. So we have

$$
\operatorname{sp}_{A}(F) \preceq\left(\rho(A), \nu_{\rho(A)}(A)-1\right) \prec \operatorname{sp}_{A}(K) .
$$

Proof of Theorem 5.1. By definition of $m_{\lambda}$ there exists a distinguished generalized eigenvector $x$ of $A$ corresponding to $\lambda$ of order $m_{\lambda}$. Clearly the vector $y=$ $(I+A)^{n-1} x$ is also a distinguished generalized eigenvector corresponding to $\lambda$ of order $m_{\lambda}$, and by Lemma $2.1 \Phi(y)$ is an $A$-invariant face. Thus, it is possible to find an $A$-invariant face of $K$ that contains in its relative interior a generalized eigenvector of $A$ corresponding to $\lambda$ of order $m_{\lambda}$. Choose $F_{m_{\lambda}}$ to be an $A$-invariant face of $K$ minimal with respect to this property. By definition, clearly we have $\operatorname{sp}_{A}\left(F_{m_{\lambda}}\right)=\left(\lambda, m_{\lambda}\right)$. We contend that $F_{m_{\lambda}}$ is $A$-invariant join-irreducible, and hence is semi-distinguished. Suppose not. Then we can find $A$-invariant faces $G_{1}, G_{2}$ properly included in $F_{m_{\lambda}}$ such that $F_{m_{\lambda}}=G_{1} \vee G_{2}$. By property (c) of Theorem 4.9(i), either $\operatorname{sp}_{A}\left(G_{1}\right)$ or $\operatorname{sp}_{A}\left(G_{2}\right)$ is equal to $\operatorname{sp}_{A}\left(F_{m_{\lambda}}\right)$, say, $\operatorname{sp}_{A}\left(G_{1}\right)$. Then, by definition of the spectral pair of a face and by Theorem $4.4, \rho_{G_{1}}=\lambda$ and $\nu_{\lambda}\left(\left.A\right|_{\text {span } G_{1}}\right)=m_{\lambda}$. Since $G_{1}$ is polyhedral, according to Tam3, Theorem 7.5(ii)], we can find in $G_{1}$ a generalized eigenvector $u$ of $A$ corresponding to $\lambda$ of order $m_{\lambda}$ such that $(A-\lambda I)^{i} u \in G_{1}$ for $i=1, \cdots, m_{\lambda}-1$. Thus, $\Phi\left((I+A)^{n-1} u\right)$ is an $A$-invariant face included in $G_{1}$, and hence properly included in $F_{m_{\lambda}}$, with the property that it contains in its relative interior a generalized eigenvector of $A$ corresponding to $\lambda$ of order $m_{\lambda}$. This contradicts the minimality of $F_{m_{\lambda}}$, and hence proves our contention for $F_{m_{\lambda}}$.

Next, in view of the polyhedrality of the cone $F_{m_{\lambda}}$, by [Tam3, Theorem 7.5(ii)] again, we can find in $F_{m_{\lambda}}$ a generalized eigenvector of $A$ corresponding to $\lambda$ of order $m_{\lambda}-1$. Then we can repeat the above argument to obtain a semi-distinguished $A$-invariant face $F_{m_{\lambda}-1}$ of $F_{m_{\lambda}}$ such that $\operatorname{sp}_{A}\left(F_{m_{\lambda}-1}\right)=\left(\lambda, m_{\lambda}-1\right)$. Continuing in this way, after a finite number of steps, we eventually arrive at our desired chain of $A$-invariant faces.

To show that our chain of $A$-invariant faces is of maximum cardinality, let $H_{1} \subseteq$ $\cdots \subseteq H_{p}$ be an arbitrary chain of distinct semi-distinguished $A$-invariant faces associated with $\lambda$. Then for each $i, 1 \leq i \leq p, \rho_{H_{i}}=\lambda$. In view of Lemma 5.2 and the definition of $m_{\lambda}$, it follows that

$$
(\lambda, 1) \preceq \operatorname{sp}_{A}\left(H_{1}\right) \prec \operatorname{sp}_{A}\left(H_{2}\right) \prec \cdots \prec \operatorname{sp}_{A}\left(H_{p}\right) \preceq\left(\lambda, m_{\lambda}\right) .
$$

Hence, $p \leq m_{\lambda}$. This shows that the chain of $A$-invariant faces (with the desired property) we have constructed is of maximum cardinality.

When $K$ is a nonpolyhedral cone, the maximum cardinality of a chain of semidistinguished $A$-invariant faces of $K$ associated with a distinguished eigenvalue $\lambda$ 
can be less than, equal to, or greater than $m_{\lambda}$, where $m_{\lambda}$ has the same meaning as before. The following examples will serve to illustrate this point and also other related points.

Example 5.3. Let $\alpha$ be a given real number with $0<\alpha<1$. Let $C$ be the closed convex set in $\mathbb{R}^{2}$ with extreme points $\left(k, \alpha^{k-1}\right)^{T}, k=1,2, \cdots$, and with recession cone $\mathbf{0}^{+} C=\left\{\lambda(1,0)^{T}: \lambda \geq 0\right\}$. Let $K$ be the proper cone of $\mathbb{R}^{3}$ given by

$$
K=\left\{\lambda\left(\begin{array}{l}
x \\
1
\end{array}\right) \in \mathbb{R}^{3}: x \in C, \lambda \geq 0\right\} \cup\left\{\lambda(1,0,0)^{T}: \lambda \geq 0\right\} .
$$

Let

$$
A=\left[\begin{array}{lll}
1 & 0 & 1 \\
0 & \alpha & 0 \\
0 & 0 & 1
\end{array}\right] .
$$

(The cone $K$ and the matrix $A$ have been considered in [T-W, Example 3.7].)

Straightforward calculations show that we have $A\left(k, \alpha^{k-1}, 1\right)^{T}=\left(k+1, \alpha^{k}, 1\right)^{T}$ for $k=1,2, \cdots$, and $A(1,0,0)^{T}=(1,0,0)^{T}$. It follows that $A \in \pi(K)$. Clearly, $\rho(A)=1$ and $\nu_{\rho(A)}(A)=2$. It is also not difficult to see that $A$ has only two nonzero $A$-invariant faces, namely $K$ and $F$, where $F=\Phi\left((1,0,0)^{T}\right)$, both of which are $A$ invariant join-irreducible, but only $\Phi\left((1,0,0)^{T}\right)$ is semi-distinguished $A$-invariant. In this case, we have $m_{\rho(A)}=1<2=\nu_{\rho(A)}(A)$. Here are two other observations we can make of this example (for the nonpolyhedral case):

(i) There does not exist a semi-distinguished $A$-invariant face $G$ of $K$ such that $\operatorname{sp}_{A}(G)=\operatorname{sp}_{A}(K)$. Indeed, $F$ is the only semi-distinguished $A$-invariant face of $K$, and we have $\operatorname{sp}_{A}(F)=(1,1) \prec(1,2)=\operatorname{sp}_{A}(K)$.

(ii) $K$ is not a semi-distinguished $A$-invariant face of itself, though it has the property that $\operatorname{sp}_{A}(K) \succ \operatorname{sp}_{A}(G)$ for all $A$-invariant faces $G$ properly included in $K$.

Example 5.4. Let $C$ be the closed convex set in $\mathbb{R}^{2}$ with recession cone $\mathbf{0}^{+} C=$ $\left\{\lambda(1,0)^{T}: \lambda \geq 0\right\}$, and with extreme points $(k(k-1) / 2, k)^{T}, k=0,1,2, \cdots$. Let $K$ be the proper cone of $\mathbb{R}^{3}$ given by

$$
K=\left\{\lambda\left(\begin{array}{l}
x \\
1
\end{array}\right) \in \mathbb{R}^{3}: x \in C, \lambda \geq 0\right\} \cup\left\{\left(\begin{array}{l}
x \\
0
\end{array}\right) \in \mathbb{R}^{3}: x \in \mathbf{0}^{+} C\right\} .
$$

Let $A=J_{3}(1)$, the $3 \times 3$ (upper triangular) elementary Jordan matrix corresponding to the eigenvalue 1. (The matrix $A$ and the cone $K$ have been considered in Tam3. Example 5.5].) It is straightforward to check that $A(1,0,0)^{T}=(1,0,0)^{T}$, and $A\left((k(k-1) / 2, k, 1)^{T}\right)=(k(k+1) / 2, k+1,1)^{T}$ for $k=0,1,2, \cdots$. It follows that $A \in \pi(K)$. Clearly, $A$ has only two nonzero $A$-invariant faces, namely $K$ and $\Phi\left((1,0,0)^{T}\right)$, both of which are semi-distinguished $A$-invariant. In this case, every nonzero vector of $K$ is a distinguished generalized eigenvector of $A$ corresponding to $\rho(A)(=1)$. Also $m_{\rho(A)}=\nu_{\rho(A)}(A)=3$, but it is impossible to find a chain of three distinct semi-distinguished $A$-invariant faces of $K$.

Example 5.5. Let $K$ be a 3-dimensional proper cone whose intersection with the hyperplane $\left\{\left(\xi_{1}, \xi_{2}, \xi_{3}\right)^{T}: \xi_{1}+\xi_{2}+\xi_{3}=1\right\}$ is a convex set $C$ lying inside the simplex $\operatorname{conv}\left\{e_{1}, e_{2}, e_{3}\right\}$, where $e_{1}, e_{2}, e_{3}$ are the standard unit vectors of $\mathbb{R}^{3}$, and with the relative boundary formed by the line segment joining $e_{1}$ and $e_{2}$, together with some strictly convex smooth curve.

Let $A=J_{2}(0) \oplus J_{1}(0)$. (Note that, in fact, $A=e_{1} e_{2}{ }^{T}$.) It is straightforward to check that $A \in \pi(K)$ and also that $K$ has three nonzero $A$-invariant faces which are 
pairwise comparable, namely, $\Phi\left(e_{1}\right), \Phi\left(e_{1}+e_{2}\right)$ and $K$ itself. Indeed, each of them is a semi-distinguished $A$-invariant face associated with $\rho(A)(=0)$. So, in this case, the cardinality of a maximum chain of semi-distinguished $A$-invariant faces associated with $\rho(A)$ is 3 , and is greater than $m_{\rho(A)}$, which is 2 . Note that we also have

$$
\operatorname{sp}_{A}(K)=(0,2)=\operatorname{sp}_{A}\left(\Phi\left(e_{1}+e_{2}\right)\right),
$$

even though the $A$-invariant face $\Phi\left(e_{1}+e_{2}\right)$ is properly included in the semidistinguished $A$-invariant face $K$.

It is also worth noting that in this example the fact that $\rho(A)=0$ is not a crucial condition. If we replace $A$ by $A+I$, the example still works.

\section{Semi-Distinguished $A$-Invariant faces}

Certainly, a natural question to ask is, when is a nonzero $A$-invariant face $F$ of $K$ semi-distinguished $A$-invariant? Clearly $F$ is a semi-distinguished $A$-invariant

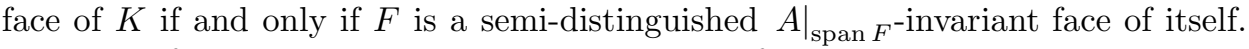
Thus, it is fundamental to consider the problem of when $K$ is a semi-distinguished $A$-invariant face of itself. We have the following result, which is motivated by the proof of Lemma 5.2.

Theorem 6.1. Let $K$ be a proper cone and let $A \in \pi(K)$. Consider the following conditions:

(a) $A^{T}$ has (up to multiples) a unique distinguished eigenvector for $K^{*}$.

(b) $K$ is a semi-distinguished $A$-invariant face of itself.

(c) $\operatorname{sp}_{A}(K) \succ \operatorname{sp}_{A}(F)$ for all $A$-invariant faces $F$ properly included in $K$.

We always have $(\mathrm{a}) \Longrightarrow(\mathrm{b})$ and $(\mathrm{a}) \Longrightarrow(\mathrm{c})$. If $K^{*}$ is a facially exposed cone, then conditions (a), (b) and (c) are equivalent.

Proof. (a) $\Longrightarrow(\mathrm{b})$. When $K^{*}$ contains a unique eigenvector of $A^{T}$, clearly $\rho(A)$ is the only distinguished eigenvalue of $A^{T}$ for $K^{*}$. In this case, by Tam3, Theorem 5.1] $K$ must contain in its interior a generalized eigenvector of $A$. So it suffices to establish the $A$-invariant join-irreducibility of $K$. Assume to the contrary that there exist $A$-invariant faces $F_{1}, F_{2}$ of $K$, both different from $K$, such that $K=F_{1} \vee F_{2}$. Then $d_{K}\left(F_{1}\right), d_{K}\left(F_{2}\right)$ are nonzero $A^{T}$-invariant faces of $K^{*}$, and hence must contain distinguished eigenvectors of $A^{T}$, say, $z_{1}$ and $z_{2}$ respectively. But

$$
d_{K}\left(F_{1}\right) \wedge d_{K}\left(F_{2}\right)=d_{K}\left(F_{1} \vee F_{2}\right)=d_{K}(K)=\{\mathbf{0}\},
$$

so the vectors $z_{1}$ and $z_{2}$ are linearly independent. This contradicts condition (a).

(a) $\Longrightarrow$ (c) by the argument used in the second half of the proof of Lemma 5.2.

Now suppose, in addition, that $K^{*}$ is a facially exposed cone.

(b) $\Longrightarrow$ (a) by the argument used in the first half of the proof of Lemma 5.2.

(c) $\Longrightarrow$ (b). By condition (c) $K$ is an $A$-invariant join-irreducible face of itself. Suppose that $K$ is not semi-distinguished $A$-invariant. Then necessarily int $K$ contains no generalized eigenvector of $A$. Hence, $A^{T}$ has a distinguished eigenvalue for $K^{*}$ other than $\rho(A)$, say $\lambda$, and let $z \in K^{*}$ be a corresponding eigenvector. Choose a distinguished eigenvector of $A^{T}$ corresponding to $\rho(A)$ that is associated with a maximal block, say $w$. We contend that $w \in d_{K} \circ d_{K^{*}}(\Phi(z))$. Take any vector $x$ from $d_{K^{*}}(\Phi(z))$. Since $z$ is an eigenvector of $A^{T}, d_{K^{*}}(\Phi(z))$ is an $A$-invariant face of $K$, different from $K$. In view of condition (c), $\operatorname{sp}_{A}(x) \preceq \operatorname{sp}_{A}\left(d_{K^{*}}(\Phi(z))\right) \prec$ $\operatorname{sp}_{A}(K)=\left(\rho(A), \nu_{\rho(A)}(A)\right)$; so by Remark 4.12 we have $\langle x, \bar{w}\rangle=0$. This proves our contention. Note that $w \notin \Phi(z)$, as $\operatorname{sp}_{A^{T}}(w)=(\rho(A), 1) \nprec(\lambda, 1)=\operatorname{sp}_{A^{T}}(z)$. 
Hence, we have $\Phi(z) \neq d_{K} \circ d_{K^{*}}(\Phi(z))$. In other words, $\Phi(z)$ is not an exposed face. This contradicts our hypothesis that $K^{*}$ is a facially exposed cone.

Our proof of Theorem $6.1,(\mathrm{c}) \Longrightarrow$ (b) actually also establishes the following positive results:

Theorem 6.2. Let $K$ be a proper cone and let $A \in \pi(K)$. Suppose that $\operatorname{sp}_{A}(K) \succ$ $\operatorname{sp}_{A}(F)$ for all $A$-invariant faces $F$ properly included in $K$. If $K$ is not a semidistinguished A-invariant face of itself, then, for any distinguished eigenvector $z$ of $A^{T}$ for $K^{*}$ corresponding to a distinguished eigenvalue other than $\rho(A), \Phi(z)$ is a non-exposed face of $K^{*}$.

Corollary 6.3. Let $A \in \pi(K)$, and suppose that $\operatorname{sp}_{A}(K) \succ \operatorname{sp}_{A}(F)$ for all $A$ invariant faces $F$ properly included in $K$. If, for any non-exposed face $G$ of $K^{*}$, the face $d_{K^{*}}(G)$ of $K$ is not $A$-invariant, then $K$ is a semi-distinguished $A$-invariant face of itself.

It is worth noting that condition (a) of Theorem 6.1 is slightly weaker than the following equivalent condition for $A^{T}$ to be $K^{*}$-irreducible:

$A^{T}$ has (up to multiples) a unique distinguished eigenvector for $K^{*}$, and this eigenvector lies in int $K^{*}$.

In general, conditions (b), (c) of Theorem 6.1 are logically independent. By Examples 5.3 and 5.5 respectively, we do not have the implications $(\mathrm{c}) \Longrightarrow$ (b) and $(\mathrm{b}) \Longrightarrow(\mathrm{c})$ (and hence also not $(\mathrm{c}) \Longrightarrow(\mathrm{a})$ and $(\mathrm{b}) \Longrightarrow(\mathrm{a})$ ).

The following result clarifies the logical relations between a number of natural conditions on a cone-preserving map that involve the concepts of semi-distinguished $A$-invariant faces, distinguished generalized eigenvectors, and spectral pairs of faces.

Theorem 6.4. Let $A \in \pi(K)$, and let $\lambda$ be a distinguished eigenvalue of $A$ for $K$. Consider the following conditions:

(a)

(i) Any A-invariant face of $K$ which contains in its relative interior a generalized eigenvector of $A$ corresponding to $\lambda$ can be expressed as a join of semi-distinguished $A$-invariant faces associated with $\lambda$.

(ii) Let $C$ denote the cone $K \cap \mathcal{N}\left((\lambda I-A)^{n}\right)$, and let $B$ be the restriction map $\left.A\right|_{\text {span } C}$. For any semi-distinguished $B$-invariant face $F$ of $C, \Phi(F)$ is a semidistinguished A-invariant face of $K$.

(b)

(i) For each nonzero A-invariant face $F$ of $K$ associated with $\lambda$, there exists a semi-distinguished $A$-invariant face $G \subseteq F$ such that $\operatorname{sp}_{A}(G)=\operatorname{sp}_{A}(F)$.

(ii) For each nonzero $A$-invariant face $F$ of $K$ associated with $\lambda$, there exists in $F$ a generalized eigenvector of $A$ corresponding to $\lambda$ of order $\nu_{\lambda}\left(\left.A\right|_{\operatorname{span} F}\right)$.

(iii) For each nonzero $A$-invariant face $F$ of $K$ associated with $\lambda, F$ is a semidistinguished $A$-invariant face, if $\operatorname{sp}_{A}(G) \prec \operatorname{sp}_{A}(F)$ for all $A$-invariant faces $G$ properly included in $F$.

(c) For any $A$-invariant face $F$ of $K$ which contains in its relative interior a generalized eigenvector of $A$ corresponding to $\lambda$, there exists a semi-distinguished $A$-invariant face $G$ included in $F$ such that $\operatorname{sp}_{A}(G)=\operatorname{sp}_{A}(F)$.

Then conditions (i), (ii) given in (a) (and also conditions (i)-(iii) given in (b)) are equivalent. Furthermore, $(\mathrm{a}) \Longrightarrow(\mathrm{c})$ and $(\mathrm{b}) \Longrightarrow(\mathrm{c})$. 
Proof. First, we show that conditions (i) and (ii) of (a) are equivalent.

(i) $\Longrightarrow$ (ii). Let $F$ be a semi-distinguished $B$-invariant face of $C$. Since $F$ contains in its relative interior a generalized eigenvector of $A$ corresponding to $\lambda$, so does $\Phi(F)$; hence, by condition (i), we can write $\Phi(F)$ as $G_{1} \vee \cdots \vee G_{k}$, where each $G_{i}$ is a semi-distinguished $A$-invariant face of $K$ associated with $\lambda$. If $k=1$, we are done. So suppose that $k \geq 2$ and also that each $G_{i}$ is properly included in $\Phi(F)$. It is straightforward to show that each $F_{i} \cap \mathcal{N}\left((\lambda I-A)^{n}\right)$ is a $B$-invariant face of $C$ properly included in $F$, and also that the join (in $C$ ) of these faces is $F$. This contradicts our assumption on $F$.

(ii) $\Longrightarrow($ i). Consider any $A$-invariant face $F$ of $K$ which contains in its relative interior a generalized eigenvector of $A$ corresponding to $\lambda$. Let $G$ denote $F \cap$ $\mathcal{N}\left((\lambda I-A)^{n}\right)$. Then $G$ is a $B$-invariant face of $C$, and $\Phi(G)=F$. Now write $G$ as the join of some $B$-invariant join-irreducible faces of $C$, say, $G_{1}, \cdots, G_{k}$. Since $\lambda$ is the only eigenvalue of $B$, clearly the faces $G_{1}, \cdots, G_{k}$ are all semi-distinguished $B$-invariant. Then $F=\Phi(G)=\Phi\left(G_{1}\right) \vee \cdots \vee \Phi\left(G_{k}\right)$, where each $\Phi\left(G_{i}\right)$ is clearly associated with $\lambda$ and, by condition (ii), is a semi-distinguished $A$-invariant face of $K$.

Next, we show that conditions (i), (ii) and (iii) of (b) are equivalent.

(i) $\Longrightarrow$ (ii). Let $F$ be an $A$-invariant face associated with $\lambda$, for which there exists, by condition (i), a semi-distinguished $A$-invariant face $G \subseteq F$ such that $\operatorname{sp}_{A}(G)=\operatorname{sp}_{A}(F)$. By definition of a semi-distinguished $A$-invariant face, there exists a generalized eigenvector $x$ of $A$ corresponding to $\rho_{G}=\rho_{F}=\lambda$ that lies in relint $G$. The order of $x$ as a generalized eigenvector of $A$ is, by definition of the spectral pair of a face and by Theorem 4.4, equal to $\nu_{\lambda}\left(\left.A\right|_{\text {span } G}\right)$, and hence to $\nu_{\lambda}\left(\left.A\right|_{\text {span } F}\right)$, as $\operatorname{sp}_{A}(G)=\operatorname{sp}_{A}(F)$. Thus, $x$ is the desired vector.

(ii) $\Longrightarrow$ (iii). The given spectral pair condition on $F$ clearly implies its $A$-invariant join-irreducibility. By condition (ii), $F$ contains at least one generalized eigenvector of $A$, say $x$, corresponding to $\rho_{F}(=\lambda)$ of $\operatorname{order} \nu_{\rho_{F}}\left(\left.A\right|_{\text {span } F}\right)$. Replacing $x$ by $(I+A)^{n-1} x$, if necessary, we may assume that $\Phi(x)$ is $A$-invariant. Since $\Phi(x) \subseteq F$ and $\operatorname{sp}_{A}(\Phi(x))=\left(\rho_{F}, \nu_{F}\left(\left.A\right|_{\text {span } F}\right)\right)=\operatorname{sp}_{A}(F)$, by the given condition on $F$, we have $\Phi(x)=F$, and hence $x \in \operatorname{relint} F$. This proves that $F$ is semi-distinguished $A$-invariant.

(iii) $\Longrightarrow$ (i). Let $F$ be a nonzero $A$-invariant face associated with $\lambda$. Choose an $A$-invariant face $G$ included in $F$ minimal with respect to the property that $\operatorname{sp}_{A}(G)=\operatorname{sp}_{A}(F)$. In view of condition (iii), $G$ must be semi-distinguished $A$ invariant. Hence, condition (i) follows.

(a) (i) $\Longrightarrow$ (c) by Theorem 4.9(i)(c).

(b) (i) $\Longrightarrow($ c). Obvious.

The proof of the implication (i) $\Longrightarrow$ (ii) in Theorem 6.4 (b) actually establishes the following result:

Remark 6.5. Given a nonzero $A$-invariant face $F$ of $K$, if there exists a semidistinguished $A$-invariant face $G$ included in $F$ such that $\operatorname{sp}_{A}(G)=\operatorname{sp}_{A}(F)$, then there exists in $F$ a generalized eigenvector of $A$ corresponding to $\rho_{F}$ of order $\nu_{\rho_{F}}\left(\left.A\right|_{\text {span } F}\right)$.

As we shall show in Example 8.1 of Section 8, the converse of Remark 6.5 does not hold. 
Some further remarks about the conditions (a), (b) and (c) of Theorem 6.4 are in order. In general, we do not have the implication $(\mathrm{a}) \Longrightarrow(\mathrm{b})$. As one can readily check, for the $A$ and $K$ of Example 5.3, with $\lambda=\rho(A)$, condition (a)(i) is satisfied, but not condition (b)(i). Whether the implication (b) $\Longrightarrow$ (a) holds or not is unknown to us. In Example 8.2 we shall show that the implications $(\mathrm{c}) \Longrightarrow(\mathrm{a})$ and (c) $\Longrightarrow(\mathrm{b})$ both do not hold.

If $K$ is a proper cone with the property that the dual cone of each of its faces is a facially exposed cone and if $A \in \pi(K)$, then according to Theorem 6.1, for any nonzero $A$-invariant face $F$ of $K, F$ is semi-distinguished $A$-invariant if and only if $\operatorname{sp}_{A}(F) \succ \operatorname{sp}_{A}(G)$ for all $A$-invariant faces $G$ properly included in $F$; thus, for all distinguished eigenvalues $\lambda$ of $A$, condition (b) (and hence also condition (c)) of Theorem 6.4 is satisfied. Clearly, proper cones $K$ all of whose nontrivial faces are polyhedral and for which $K^{*}$ are facially exposed cones have the property. Such cones include all polyhedral cones, proper cones of $\mathbb{R}^{3}$ whose dual cones are facially exposed, and also strictly convex smooth cones. [Recall that a proper cone $K$ is said to be strictly convex if each of its nontrivial faces is one-dimensional, and is smooth if for each nonzero boundary vector $x$ of $K$, $\operatorname{dim} d_{K}(\Phi(x))=1$. It can be shown that the class of strictly convex cones whose dual cones are facially exposed is the same as the class of strictly convex smooth cones.].

Perfect cones form another interesting class with the above-mentioned property. Following [Bar1, we call a proper cone a perfect cone if each of its faces is self-dual in its own linear span. Examples of perfect cones include the nonnegative orthant $\mathbb{R}_{+}^{n}$, the $n$-dimensional ice-cream cone $K_{n}:=\left\{\left(\xi_{1}, \cdots, \xi_{n}\right)^{T}: \xi_{1} \geq\left(\xi_{2}{ }^{2}+\cdots+\xi_{n}{ }^{2}\right)^{1 / 2}\right\}$, and the cone of $n \times n$ positive semi-definite hermitian (or real symmetric) matrices. An equivalent definition for $K$ to be a perfect cone is that $K$ is self-dual, and for each face $F$ of $K$ the orthogonal projection $p_{F}$ of $\mathbb{R}^{n}$ onto span $F$ belongs to $\pi(K)$ (see [Bar1, Proposition 1]). So a perfect cone is an orthogonal projectionally exposed, and hence projectionally exposed, cone (see $[\mathrm{Su}-\mathrm{T}]$ for definitions). But by [Su-T, Corollary 4.4 and Lemma 2.2$]$ each face of a projectionally exposed cone is a facially exposed cone in its own right. Hence, each face of a perfect cone is itself a facially exposed self-dual cone.

For each nonnegative integer $n$, we use $P(n)$ to denote the cone of all real polynomials of degree not exceeding $n$ that are nonnegative on the closed interval $[0,1]$. In $[\mathrm{B}-\mathrm{T}]$ it is proved that $P(n)^{*}$ is always a facially exposed cone, and also that each nonzero face of $P(n)$ is linearly isomorphic with $P(m)$ for some nonnegative integer $m \leq n$. Thus, the cone $P(n)$ also serves as another interesting example of a proper cone with the property that the dual cone of each of its faces is a facially exposed cone.

To sum up our preceding discussions, we have the following result.

Theorem 6.6. (i) Let $K$ be a proper cone with the property that the dual cone of each of its faces is a facially exposed cone, and let $A \in \pi(K)$. Then for any nonzero $A$-invariant face $F$ of $K, F$ is semi-distinguished $A$-invariant if and only if $\operatorname{sp}_{A}(G) \prec \operatorname{sp}_{A}(F)$ for all $A$-invariant faces $G$ properly included in $F$. Consequently, for all distinguished eigenvalues $\lambda$ of A, condition (b) (and hence (c)) of Theorem 6.4 is satisfied.

(ii) A proper cone $K$ has the property given in the hypothesis of part (i), if it fulfills one of the following conditions: 
(a) $K^{*}$ is a facially exposed cone, and all nontrivial faces of $K$ are polyhedral (which is the case if $K$ is polyhedral, or is a strictly convex smooth cone).

(b) $K$ is a perfect cone.

(c) $K$ equals $P(n)$ for some nonnegative integer $n$.

Note that we could have formulated the last part of Theorem 6.6 (i) without mentioning the distinguished eigenvalues of $A$. Indeed, the statement that, for all distinguished eigenvalues $\lambda$ of $A$, condition (b) (ii) of Theorem 6.4 is satisfied amounts to saying that for each nonzero $A$-invariant face $F$ of $K$, there exists in $F$ a distinguished generalized eigenvector of $A$ corresponding to $\rho_{F}$ of order $\nu_{\rho_{F}}\left(\left.A\right|_{\text {span } F}\right)$. We leave it to the reader to give similar reformulations for the statement that conditions (b) (ii), (iii) or (c) of Theorem 6.4 are satisfied for all distinguished eigenvalues $\lambda$ of $A$.

Theorem 6.7. Let $A \in \pi(K)$, where $K$ is a polyhedral cone or a strictly convex cone. Then for any distinguished eigenvalue $\lambda$ of A, condition (a) of Theorem 6.4 is satisfied.

The polyhedral case of Theorem 6.7 is covered by Lemma 6.8. The proof for a strictly convex cone will follow from Lemma 6.10.

Lemma 6.8. Let $K$ be a polyhedral cone. Let $A \in \pi(K)$, and let $\lambda$ be a distinguished eigenvalue of $A$ for $K$. Let $C$ denote the cone $K \cap \mathcal{N}\left((\lambda I-A)^{n}\right)$, and let $B$ denote the restriction map $\left.A\right|_{\text {span } C}$. Then for any semi-distinguished $B$-invariant face $F$ of $C, \Phi(F)$ is a semi-distinguished A-invariant face of $K$.

Proof. Since $K$ is polyhedral, by Theorem 6.6 it suffices to show that $\operatorname{sp}_{A}(G) \prec$ $\operatorname{sp}_{A}(\Phi(F))$ for any $A$-invariant face $G$ properly included in $\Phi(F)$. If $\rho_{G}<\lambda$, clearly there is no problem. So, suppose that $\rho_{G}=\lambda$. By Theorem 6.6 again, condition (b) (i) of Theorem 6.4 is satisfied. Hence, there exists a semi-distinguished $A$-invariant face $H$ included in $G$ such that $\operatorname{sp}_{A}(H)=\operatorname{sp}_{A}(G)$. Then $H \cap \mathcal{N}\left((\lambda I-A)^{n}\right)$ is a $B$-invariant face of $C$ properly included in $F$. Since $F$ is a semi-distinguished $B$-invariant face of the polyhedral cone $C$, by Theorem 6.6 again we have

$$
\operatorname{sp}_{B}\left(H \cap \mathcal{N}\left((\lambda I-A)^{n}\right)\right) \prec \operatorname{sp}_{B}(F) .
$$

Now relint $F \subseteq \operatorname{relint} \Phi(F)$ and $B$ is a restriction of $A$; so by the definition of the spectral pair of a face, we have $\operatorname{sp}_{B}(F)=\operatorname{sp}_{A}(\Phi(F))$. But $H$ contains in its relative interior a generalized eigenvector of $A$ corresponding to $\lambda$, so for a similar reason, we also have $\operatorname{sp}_{B}\left(H \cap \mathcal{N}\left((\lambda I-A)^{n}\right)\right)=\operatorname{sp}_{A}(H)$. Hence, the desired inequality $\operatorname{sp}_{A}(G) \prec \operatorname{sp}_{A}(\Phi(F))$ follows.

For completeness, we also note that the converse of Lemma 6.8 always holds, even without the polyhedrality assumption on $K$. The proof is straightforward.

Remark 6.9. Let $A \in \pi(K)$ and let $\lambda$ be a distinguished eigenvalue of $A$ for $K$. Let $C$ denote the cone $K \cap \mathcal{N}\left((\lambda I-A)^{n}\right)$ and let $B$ denote the restriction map $\left.A\right|_{\text {span } C}$. For any face $F$ of $C$, if $\Phi(F)$ is a semi-distinguished $A$-invariant face of $K$, then $F$ is a semi-distinguished $B$-invariant face of $C$.

Lemma 6.10. Let $K$ be a strictly convex cone, and let $A \in \pi(K)$. For any $A$ invariant face $F$ of $K$, we have $\operatorname{sp}_{A}(F) \succ \operatorname{sp}_{A}(G)$ for all $A$-invariant faces $G$ properly included in $F$, whenever $F$ is a semi-distinguished $A$-invariant face of $K$. 
Proof. It is clear that each nontrivial $A$-invariant face of $K$, being an extreme ray of $K$, is a distinguished $A$-invariant face and satisfies trivially the desired property involving spectral pairs. So it suffices to consider the case $F=K$. If $\nu_{\rho(A)}(A)>1$, then for any nonzero $A$-invariant face $G$ properly included in $K$ we have

$$
\operatorname{sp}_{A}(K)=\left(\rho(A), \nu_{\rho(A)}(A)\right) \succ(\rho(A), 1) \succeq\left(\rho_{G}, 1\right)=\operatorname{sp}_{A}(G),
$$

where the last equality holds as $G$ is an extreme ray. So suppose that $\nu_{\rho(A)}(A)=1$. Then the generalized eigenvector of $A$ (corresponding to $\rho(A)$ ) that lies in int $K$ is in fact an eigenvector. Hence, condition (d) of Theorem 4.11 is satisfied; that is, $K$ is a distinguished $A$-invariant face of itself, and so we also have $\operatorname{sp}_{A}(K) \succ \operatorname{sp}_{A}(G)$ for any $A$-invariant face $G$ properly included in $K$.

We now give the proof of Theorem 6.7 for the strictly convex case:

Clearly it suffices to show that if $K$ contains in its interior a generalized eigenvector of $A$, then $K$ can be expressed as a join of semi-distinguished $A$-invariant faces associated with $\rho(A)$. If $\nu_{\rho(A)}(A)>1$ then, as shown in the proof of Lemma 6.10 , we have $\operatorname{sp}_{A}(K) \succ \operatorname{sp}_{A}(G)$ for all $A$-invariant faces $G$ properly included in $K$; hence, $K$ is $A$-invariant join-irreducible, and since $K$ contains in its interior a generalized eigenvector of $A, K$ is itself a semi-distinguished $A$-invariant face. When $\nu_{\rho(A)}(A)=1$, then either $K$ is a distinguished $A$-invariant face of itself, or $\operatorname{dim}(K \cap \mathcal{N}(\rho(A) I-A)) \geq 2$. In the former case, we are done. In the latter case, since $K$ is strictly convex, clearly $K$ can be expressed as the join of two $A$-invariant extreme rays, and hence of two $A$-invariant distinguished faces, associated with $\rho(A)$.

For completeness, we also include the following interesting result on a smooth cone.

Lemma 6.11. Let $K$ be a smooth cone, and let $A \in \pi(K)$.

(i) If $\nu_{\rho(A)}(A)>1$, then $K$ contains in its interior a generalized eigenvector of A.

(ii) There always exists in $K$ a generalized eigenvector of $A$ of order $\nu_{\rho(A)}(A)$ corresponding to $\rho(A)$.

(iii) If $\operatorname{sp}_{A}(K) \succ \operatorname{sp}_{A}(G)$ for all $A$-invariant faces $G$ properly included in $K$, then $K$ is a semi-distinguished A-invariant face of itself.

Proof. (i). Assume to the contrary that $K$ does not contain in its interior a generalized eigenvector of $A$. By [Tam3 Theorem 5.1] $A^{T}$ has a distinguished eigenvalue $\lambda$ other than $\rho(A)$. Let $w \in K^{*}$ be a corresponding eigenvector. Choose from $K$ an eigenvector $x$ of $A$ corresponding to $\rho(A)$, which is associated with a maximal Jordan block (see Remark 4.2). Also choose from $K^{*}$ an eigenvector $z$ of $A^{T}$ corresponding to $\rho(A)$, which is again associated with a maximal Jordan block. Since $x$ and $w$ are respectively eigenvectors of $A$ and $A^{T}$ corresponding to distinct eigenvalues, $x$ and $w$ are mutually orthogonal. Because the size of a maximal Jordan block of $A$ corresponding to $\rho(A)$, i.e. $\nu_{\rho(A)}(A)$, is greater than 1 , the vectors $x$ and $z$ are also mutually orthogonal. Thus $x$ is orthogonal to two linearly independent vectors of $K^{*}$. This contradicts the smoothness of $K$ at $x$.

(ii). If $\nu_{\rho(A)}(A)=1$, clearly there is no problem. If $\nu_{\rho(A)}(A)>1$, our assertion follows from part (i).

(iii). If $\nu_{\rho(A)}(A)=1$, then, by the spectral pair condition on $K$, clearly $K$ is a distinguished $A$-invariant face of itself. Otherwise, by part (i) $K$ contains in its interior a generalized eigenvector of $A$. And since the spectral pair condition 
on $K$ implies the $A$-invariant join-irreducibility of $K$, it follows that $K$ is a semidistinguished $A$-invariant face of itself.

The following example shows that parts (i)-(iii) of Lemma 6.11 all do not hold, if in the hypothesis on $K$, "smooth" is replaced by "strictly convex". Consequently, the converse of Lemma 6.10 also does not hold.

Example 6.12. Let $\alpha$ be a positive real number less than 1 . Let $f$ be a real-valued function defined on the real line that possesses the following properties:

(i) $f$ is a differentiable convex function;

(ii) $\lim _{\xi \rightarrow-\infty} f(\xi)=\infty$;

(iii) $\lim _{\xi \rightarrow \infty} f(\xi)=-\infty$;

(iv) $\lim _{\xi \rightarrow \infty} f^{\prime}(\xi)=0$; and

(v) For each $\xi \in \mathbb{R}$, we have $f(\xi+1) \leq \alpha f(\xi)$.

For instance, if $e^{-1}<\alpha<1$ and $f$ is given by

$$
f(\xi)=\left\{\begin{array}{lll}
-\log (\xi+1) & \text { for } \quad \xi \geq 0, \\
\exp (-\xi)-1 & \text { for } \quad \xi<0,
\end{array}\right.
$$

then one can readily verify that $f$ possesses properties (i)-(v).

Denote the epigraph of $f$, i.e. the set $\left\{\left(\xi_{1}, \xi_{2}\right)^{T} \in \mathbb{R}^{2}: \xi_{2} \geq f\left(\xi_{1}\right)\right\}$, by epi $f$. Since $f$ is a continuous convex function, epi $f$ is a closed convex set. By the convexity of $f$ and property (v), for any $\xi \in \mathbb{R}$, we have, $f^{\prime}(\xi) \leq f(\xi+1)-f(\xi) \leq$ $-(1-\alpha) f(\xi)$. In view of property (ii) it follows that $\lim _{\xi \rightarrow-\infty} f^{\prime}(\xi)=-\infty$. The latter condition implies that the recession cone $\mathbf{0}^{+}$(epi $f$ ) cannot contain a vector of the form $\left(\nu_{1}, \nu_{2}\right)^{T}$ with $\nu_{1}<0$. Similarly, property (iv) implies that $\mathbf{0}^{+}$(epi $f$ ) cannot contain a vector of the form $\left(\nu_{1}, \nu_{2}\right)^{T}$ with $\nu_{2}<0$. Clearly, $(0,1)^{T} \in \mathbf{0}^{+}($epi $f)$. Also, we have, $(1,0)^{T} \in \mathbf{0}^{+}$(epi $\left.f\right)$; otherwise, $f$ is not one-to-one and property (iii) cannot be met. Thus, $\mathbf{0}^{+}$(epi $f$ ) equals $\mathbb{R}_{+}^{2}$.

Let $K$ be the proper cone of $\mathbb{R}^{3}$ whose dual cone $K^{*}$ is given by

$$
K^{*}=\left\{\lambda\left(\xi_{1}, \xi_{2}, 1\right)^{T} \in \mathbb{R}^{3}: \lambda \geq 0,\left(\xi_{1}, \xi_{2}\right)^{T} \in \operatorname{epi}(f)\right\} \cup\left(\mathbf{0}^{+}(\text {epi } f) \times\{0\}\right) .
$$

Since $f$ is a differentiable function, $K^{*}$ is smooth at each nonzero boundary vector of the form $\lambda\left(\xi_{1}, \xi_{2}, 1\right)^{T}$. To show that $K^{*}$ is smooth at $(1,0,0)^{T}$, let $\left(\eta_{1}, \eta_{2}, \eta_{3}\right)^{T}$ be a nonzero vector of $K$ which is orthogonal to $(1,0,0)^{T}$. Clearly, $\eta_{1}=0$. Since $(0,1,0)^{T} \in K^{*}, \eta_{2} \geq 0$. If $\eta_{2}>0$, then in view of property (iii), we can find some $\xi \in \mathbb{R}$ such that the inner product between $\left(\eta_{1}, \eta_{2}, \eta_{3}\right)^{T}$ and $(\xi, f(\xi), 1)^{T}$ is negative, which is a contradiction. It follows that (up to multiples) $(0,0,1)^{T}$ is the only vector of $K$ orthogonal to $(1,0,0)^{T}$. Similarly, we can show that $(0,0,1)^{T}$ is also the only vector of $K$ orthogonal to $(0,1,0)^{T}$. This shows that $K^{*}$ is a smooth cone. Hence, $K$ is a strictly convex cone.

Now let

$$
A=\left[\begin{array}{lll}
1 & 0 & 0 \\
0 & \alpha & 0 \\
1 & 0 & 1
\end{array}\right] .
$$

By property (v) one readily checks that $A^{T} \in \pi\left(K^{*}\right)$; hence, $A \in \pi(K)$. Clearly, $\nu_{\rho(A)}(A)=2$. Note that $K$ does not contain a generalized eigenvector of $A$ corresponding to $\rho(A)$ of order two, because any such vector is necessarily orthogonal 
to the vector $(0,1,0)^{T}$ of $K^{*}$, contradicting what we have found above. It is now clear that $K$ is not a semi-distinguished $A$-invariant face of itself, and also that we have $\operatorname{sp}_{A}(K) \succ \operatorname{sp}_{A}(G)$ for all $A$-invariant faces $G$ properly included in $K$.

\section{Extensions of two NONNEGATIVE Matrix RESUlts}

Besides Theorem B (mentioned in Section 1), the following is another early result on the combinatorial spectral theory of a nonnegative matrix:

Theorem $\mathbf{C}\left(\underline{\mathrm{Schn1}}\right.$, Theorem 3]). If $P$ is a nonnegative matrix, then $\nu_{\rho(P)}(P)=$ 1 if and only if there are no comparable basic classes of $P$.

It is clear that Theorem C follows as a special case of Theorem A, the Rothblum index theorem.

For a general cone-preserving map, we have the following result related to Theorem C.

Theorem 7.1. Let $A \in \pi(K)$, and let $\lambda$ be a distinguished eigenvalue of $A$ for $K$. Denote by $m_{\lambda}$ the maximal order of distinguished generalized eigenvectors of $A$ corresponding to $\lambda$. Consider the following conditions:

(a) $m_{\lambda}=1$.

(b) Any two distinct semi-distinguished A-invariant faces of $K$ associated with $\lambda$ are noncomparable.

(i) We always have (a) $\Longrightarrow$ (b).

(ii) When condition (c) (and hence also when condition (a) or (b)), of Theorem 6.4 is satisfied, conditions (a) and (b) are equivalent.

Proof. (i). It is clear that any two distinct distinguished $A$-invariant faces of $K$ associated with $\lambda$ are noncomparable. So it suffices to show that, when $m_{\lambda}=1$, any semi-distinguished $A$-invariant face associated with $\lambda$ is distinguished $A$-invariant. Indeed, if $F$ is a semi-distinguished $A$-invariant face associated with $\lambda$, then by definition $F$ is $A$-invariant join-irreducible and contains in its relative interior a generalized eigenvector of $A$ corresponding to $\lambda$. Since $m_{\lambda}=1$, this generalized eigenvector is necessarily an eigenvector. Hence, by Theorem $4.11 F$ must be a distinguished $A$-invariant face of $K$ associated with $\lambda$. This establishes (b).

(ii). It suffices to establish the implication (b) $\Longrightarrow$ (a). Assume to the contrary that condition (b) is satisfied, but not condition (a). Then $m_{\lambda}>1$. Let $x$ be a distinguished generalized eigenvector of $A$ corresponding to $\lambda$ of order $m_{\lambda}$. Then $F=\Phi\left((I+A)^{n-1} x\right)$ is an $A$-invariant face that contains in its relative interior a generalized eigenvector of $A$ corresponding to $\lambda$. Furthermore, $\operatorname{sp}_{A}(F)=\left(\lambda, m_{\lambda}\right)$. By condition (c) of Theorem 6.4 there exists a semi-distinguished $A$-invariant face $G$ included in $F$ such that $\operatorname{sp}_{A}(G)=\operatorname{sp}_{A}(F)$. Now choose any extremal distinguished eigenvector $u$ of $A$ corresponding to $\lambda$ that lies in $G$. Then by Theorem $4.11 \Phi(u)$ is a distinguished, and hence semi-distinguished, $A$-invariant face associated with $\lambda$ which is properly included in $G$. This contradicts condition (b).

Later, by Example 8.1 we shall show that for the conditions (a), (b) of Theorem 7.1 , the implication $(\mathrm{b}) \Longrightarrow(\mathrm{a})$ is not valid in general.

Relative to Theorem B we have the following result.

Theorem 7.2. Let $A \in \pi(K)$, and let $\lambda$ be a distinguished eigenvalue of $A$ for $K$. Consider the following conditions:

(a) $\operatorname{dim} \mathcal{N}(\lambda I-A)=1$. 
(b) Any A-invariant face which contains in its relative interior a generalized eigenvector of $A$ corresponding to $\lambda$ is semi-distinguished $A$-invariant.

(c) Any two A-invariant faces of $K$ each containing in its relative interior a generalized eigenvector of $A$ corresponding to $\lambda$ are comparable.

(d) Any two semi-distinguished A-invariant faces of $K$ associated with $\lambda$ are comparable.

(e) $\operatorname{dim} \operatorname{span}[\mathcal{N}(\lambda I-A) \cap K]=1$.

(i) We always have the implications (a) $\Longrightarrow(\mathrm{b}) \Longrightarrow(\mathrm{c}) \Longrightarrow(\mathrm{d}) \Longrightarrow(\mathrm{e})$.

(ii) When condition (a) of Theorem 6.4 is satisfied, conditions (b), (c) and (d) are equivalent.

(iii) When $K$ is polyhedral, conditions (b), (c) and (d) are equivalent. If, in addition, $\lambda=\rho(A)$, then condition (a) is also another equivalent condition.

Proof. (i).

(a) $\Longrightarrow(\mathrm{b})$. We are required to show that if $F$ is any $A$-invariant face of $K$ that contains in its relative interior a generalized eigenvector of $A$ corresponding to $\lambda$, then $F$ is $A$-invariant join-irreducible. By considering the restriction map $\left.A\right|_{\text {span } F}$ instead of $A$, we may henceforth take $F$ to be $K$ and $\lambda$ to be $\rho(A)$. Let $G$ be any $A$-invariant face of $K$ properly included in $K$. We are going to show that $\operatorname{sp}_{A}(G) \prec \operatorname{sp}_{A}(K)$. Once this is done, the $A$-invariant join-irreducibility of $K$ will follow.

Now $\operatorname{span} G$ is an $A$-invariant subspace of $\mathbb{R}^{n}$. If $\operatorname{span} G$ contains a generalized eigenvector of $A$ corresponding to $\rho(A)$ of order $\nu_{\rho(A)}(A)$, then span $G$ will include $\mathcal{N}\left((\rho(A) I-A)^{n}\right)$, as the Jordan form of $A$ has only one block corresponding to $\rho(A)$. Since $G=K \cap \operatorname{span} G$ and $\mathcal{N}\left((\rho(A) I-A)^{n}\right)$ meets the interior of $K$, it will follow that $G$ contains an interior vector of $K$ and hence is equal to $K$, which is a contradiction. We just show that if $\rho(A)$ is an eigenvalue of $\left.A\right|_{\text {span } G}$ then its index is less than $\nu_{\rho(A)}(A)$. Now it is clear that we have $\operatorname{sp}_{A}(G) \prec \operatorname{sp}_{A}(K)$.

(b) $\Longrightarrow(\mathrm{c})$. Assume to the contrary that there exist two noncomparable $A$ invariant faces of $K$, say $F_{1}, F_{2}$, each containing in its relative interior a generalized eigenvector of $A$ corresponding to $\lambda$. Then $F_{1} \vee F_{2}$ is an $A$-invariant face with the same property, but is not semi-distinguished $A$-invariant. This contradicts condition (b).

$(\mathrm{c}) \Longrightarrow(\mathrm{d})$. Obvious.

(d) $\Longrightarrow(\mathrm{e})$. If $\operatorname{dim}[\mathcal{N}(\lambda I-A) \cap K] \geq 2$, choose any two distinct extreme vectors $x_{1}, x_{2}$ of the cone $\mathcal{N}(\lambda I-A) \cap K$. Then $\Phi\left(x_{1}\right)$ and $\Phi\left(x_{2}\right)$ are distinguished, and hence semi-distinguished, noncomparable $A$-invariant faces of $K$ associated with $\lambda$. This violates condition (d).

(ii). To prove the equivalence of conditions (b), (c), (d), it suffices to establish the implication $(\mathrm{d}) \Longrightarrow(\mathrm{b})$. Let $F$ be an $A$-invariant face which contains in its relative interior a generalized eigenvector of $A$ corresponding to $\lambda$. By condition (a)(i) of Theorem 6.4 we can write $F$ as $F_{1} \vee \cdots \vee F_{k}$, where each $F_{i}$ is a semi-distinguished $A$ invariant face associated with $\lambda$. We may assume that there are no redundant terms in this representation of $F$. If $k \geq 2$, then $F_{1}$ and $F_{2}$ will be two noncomparable semi-distinguished $A$-invariant faces associated with $\lambda$, contradicting condition (d). Hence we have $k=1$, and $F$ is semi-distinguished $A$-invariant.

(iii). When $K$ is polyhedral, by Theorem 6.7 condition (a) of Theorem 6.4 is satisfied. Hence, by part (ii), conditions (b), (c) and (d) are equivalent. 
Now suppose, in addition, that $\lambda=\rho(A)$. To prove the equivalence of conditions (a)-(d), it remains to establish the implication (d) $\Longrightarrow$ (a).

Suppose that condition (d) is satisfied. Clearly an equivalent condition for (a) is that the geometric multiplicity of $\rho(A)$ as an eigenvalue of $\left.A\right|_{\mathcal{N}\left((\rho(A) I-A)^{n}\right)}$ is one. Since $K$ is polyhedral, $\mathcal{N}\left((\rho(A) I-A)^{n}\right)$ has a basis that consists of vectors of $K$ (see [Tam3, Theorem 7.5]); so $\mathcal{N}\left((\rho(A) I-A)^{n}\right) \cap K$ is a proper cone of $\mathcal{N}\left((\rho(A) I-A)^{n}\right)$. Denote by $B$ the restriction of $A$ to $\mathcal{N}\left((\rho(A) I-A)^{n}\right)$ and by $C$ the cone $\mathcal{N}\left((\rho(A) I-A)^{n}\right) \cap K$. If $G_{1}, G_{2}$ are semi-distinguished $B$ invariant faces of $C$, then by Lemma $6.8 \Phi\left(G_{1}\right)$ and $\Phi\left(G_{2}\right)$ are semi-distinguished $A$-invariant faces of $K$ and are comparable according to condition (d). But $G_{i}=$ $\mathcal{N}\left((\rho(A) I-A)^{n}\right) \cap \Phi\left(G_{i}\right)$ for $i=1,2$; hence $G_{1}$ and $G_{2}$ are also comparable. This shows that $B[\in \pi(C)]$ also satisfies the condition corresponding to condition (d). Therefore, by considering $B$ instead of $A$, we may, henceforth, assume that $\rho(A)$ is the only eigenvalue of $A$.

Since $K$ is polyhedral, by Theorem 6.7, condition (a)(i) of Theorem 6.4 is satisfied; hence, any nonzero $A$-invariant face of $K$ (which necessarily contains in its relative interior a generalized eigenvector of $A$ corresponding to $\rho(A))$ is the join of semi-distinguished $A$-invariant faces of $K$ (associated with $\rho(A)$ ). But according to condition (d), any two semi-distinguished $A$-invariant faces of $K$ are comparable. It follows that each nonzero $A$-invariant face of $K$ is semi-distinguished. In particular, $K$ is a semi-distinguished $A$-invariant face of itself. Hence, by Theorem 6.1 $A^{T}$ has (up to multiples) a unique distinguished eigenvector for $K^{*}$, say $w$. Indeed, as noted in the proof of Lemma 5.2, in this case $A^{T}$ has precisely one maximal Jordan block corresponding to $\rho(A)$, and $w$ is associated with this maximal block. Furthermore, since $\rho(A)$ is the only eigenvalue of $A$, we have $(\operatorname{span}\{w\})^{\perp}=E_{\nu-1}$, where $E_{\nu-1}$ is the space of generalized eigenvectors of $A$ (corresponding to $\rho(A)$ ) of order less than or equal to $\nu_{\rho(A)}(A)-1$, together with the zero vector. We contend that $\Phi(w)$ is an extreme ray of $K^{*}$.

To show this, choose a nonzero extreme vector $z$ of $\Phi(w)$. Then we have

$$
(\rho(A), 1)=\operatorname{sp}_{A^{T}}(\Phi(w)) \succeq \operatorname{sp}_{A^{T}}(\Phi(z)) \succeq(\rho(A), 1),
$$

where the last inequality follows from the assumption that $\rho(A)$ is the only eigenvalue of $A$. Hence, $\operatorname{sp}_{A^{T}}(\Phi(z))=(\rho(A), 1)$, and so $z$ is an eigenvector of $A^{T}$. It follows that $z$ is a multiple of $w$. This proves our contention.

Since $K$ is polyhedral and $\Phi(w)$ is an extreme ray of $K^{*}, d_{K^{*}}(\Phi(w))$ must be a cone of dimension $n-1$ (for reference, see Tam1, Theorem 3]), and hence is a proper cone of $E_{\nu-1}$; thus we can write $\left.A\right|_{E_{\nu-1}} \in \pi\left(d_{K^{*}}(\Phi(w))\right)$. Now let the Jordan form of $A$ be

$$
J_{i_{1}}(\rho(A)) \oplus J_{i_{2}}(\rho(A)) \oplus \cdots \oplus J_{i_{k}}(\rho(A)),
$$

where $k \geq 1$, and $i_{1}>i_{2} \geq \cdots \geq i_{k}$ if $k \geq 2$. Then it is not difficult to show that the Jordan form of $\left.A\right|_{E_{\nu-1}}$ is

$$
J_{i_{1}-1}(\rho(A)) \oplus J_{i_{2}}(\rho(A)) \oplus J_{i_{3}}(\rho(A)) \oplus \cdots \oplus J_{i_{k}}(\rho(A)) .
$$

Note that $d_{K^{*}}(\Phi(w))$ is itself a polyhedral cone, and also that $\left.A\right|_{E_{\nu-1}}$ inherits from $A$ the condition corresponding to condition (d). So, applying our above argument with $\left.A\right|_{E_{\nu-1}}$ in place of $A$, we see that the Jordan form of $\left.A\right|_{E_{\nu-1}}$ also has precisely one maximal block. Suppose $k \geq 2$. If $i_{1}-1=i_{2}$, we already obtain a contradiction. Otherwise, our argument shows that $\left.A\right|_{E_{\nu-2}}$, where $E_{\nu-2}$ denotes the space of 
generalized eigenvectors of $A$ of order less than or equal to $\nu_{\rho(A)}(A)-2$, together with the zero vector, belongs to $\pi(G)$ for some $A$-invariant face $G$ of $K$ of dimension $n-2$. Furthermore, $\left.A\right|_{E_{\nu-2}}$ also has precisely one maximal block in its Jordan form, which is

$$
J_{i_{1}-2}(\rho(A)) \oplus J_{i_{2}}(\rho(A)) \oplus \cdots \oplus J_{i_{k}}(\rho(A)) .
$$

If $i_{1}-2=i_{2}$, we again arrive at a contradiction. Otherwise, we repeat the process and obtain a contradiction after a finite number of steps. This shows that $k=1$, i.e. $A$ has precisely one Jordan block (corresponding to $\rho(A)$ ), as desired.

In addition to the conditions of Theorem 7.2 one may also consider the following condition:

(f) Any two $A$-invariant faces of $K$ associated with $\lambda$ are comparable.

Clearly, (f) implies condition (c) of Theorem 7.2. On the other hand, (f) is logically independent of condition (a) of Theorem 7.2. To see why (a) does not imply (f), consider the $3 \times 3$ nonnegative matrix $A$ given by

$$
A=\left[\begin{array}{lll}
1 & 1 & 1 \\
0 & 0 & 0 \\
0 & 0 & 0
\end{array}\right] .
$$

Take $\lambda=\rho(A)$. Clearly $\rho(A)=1$, and the geometric multiplicity of 1 as an eigenvalue of $A$ is 1 . However, $\Phi\left(e_{1}+e_{2}\right)$ and $\Phi\left(e_{1}+e_{3}\right)$ are noncomparable $A$ invariant faces of $\mathbb{R}_{+}^{3}$ associated with 1 . So, in this case, condition (a) of Theorem 7.2 is satisfied, but not condition (f). Example 5.5 can be used to show that the implication (f) $\Longrightarrow(\mathrm{a})$ also does not hold. It is not clear to us whether condition (f) is sufficient for condition (b) of Theorem 7.2.

Now we give some further remarks in concern with conditions (a)-(e) of Theorem 7.2 .

When $\lambda=\rho(A)$ and $A$ is a nonnegative matrix, by Theorem 7.2 (iii) conditions (a)-(d) are all equivalent. However, condition (e) is strictly weaker. To see this, consider the following $4 \times 4$ nilpotent nonnegative matrix:

$$
A=\left[\begin{array}{llll}
0 & 1 & 1 & 0 \\
0 & 0 & 0 & 1 \\
0 & 0 & 0 & 1 \\
0 & 0 & 0 & 0
\end{array}\right] .
$$

In this case, each of the singletons $\{1\},\{2\},\{3\}$ and $\{4\}$ is a class, indeed a basic class, of $A$. Also, $\{1\}$ is the only distinguished class of $A$ (associated with 0 ). By the Frobenius-Victory theorem, we have $\operatorname{dim}\left(\mathcal{N}(A) \cap \mathbb{R}_{+}^{n}\right)=1$; that is, condition (e) is satisfied for $\lambda=0$. (In fact, in this case, for a similar reason, the dual condition $\operatorname{dim}\left(\mathcal{N}\left(A^{T}\right) \cap \mathbb{R}_{+}^{n}\right)=1$ is also satisfied.) However, condition (a) is not satisfied for $\lambda=0$, because $\operatorname{dim} \mathcal{N}(A)=4-\operatorname{rank}(A)=2$.

In Section 8 we shall give examples which show that the missing implications of Theorem 7.2 all do not hold in general.

The following example shows that when $\lambda<\rho(A)$, condition (b) of Theorem 7.2 and the corresponding condition with $A$ replaced by $A^{T}$ and $K$ replaced by $K^{*}$ together do not imply condition (a), not even for the nonnegative matrix case, assuming that $\lambda$ is a distinguished eigenvalue of $A$ for $K$ and also of $A^{T}$ for $K^{*}$. 
Example 7.3. Consider the following $4 \times 4$ nonnegative matrix:

$$
A=\left[\begin{array}{llll}
0 & 0 & 0 & 0 \\
1 & 1 & 1 & 0 \\
1 & 1 & 2 & 0 \\
1 & 1 & 1 & 0
\end{array}\right] .
$$

In this case $A$ has precisely three classes which form a chain, namely, $\{4\},\{2,3\}$ and $\{1\} ;\{2,3\}$ being a basic class, and $\{1\}$ and $\{4\}$ being nonbasic classes both associated with 0 . Clearly $\mathbb{R}_{+}^{4}$ has precisely three nonzero $A$-invariant faces, namely, $\Phi\left(e_{4}\right), \Phi\left(e_{2}+e_{3}+e_{4}\right)$ and $\mathbb{R}_{+}^{4}$ itself. Also, $\Phi\left(e_{4}\right)$ is the only $A$-invariant face of $\mathbb{R}_{+}^{4}$ associated with 0 , indeed, a semi-distinguished $A$-invariant face; thus condition (b) of Theorem 7.2 is satisfied by $A$ for $\lambda=0$. For a similar reason, $A^{T}$ also satisfies the corresponding condition. However, condition (a) of Theorem 7.2 is not satisfied, because we have $\operatorname{dim} \mathcal{N}(A)=4-\operatorname{rank} A=2$.

In the nonpolyhedral case, even when $K$ is the $n$-dimensional ice-cream cone $K_{n}$ (which is a perfect cone, and possesses many nice properties as described in Theorem 6.6) and $\lambda=\rho(A)$, condition (b) of Theorem 7.2 and the corresponding condition for $A^{T}$ together do not guarantee condition (a). For instance, take $A$ to be $y z^{T}$, where $y$ and $z$ are any two mutually orthogonal nonzero vectors that lie in $\partial K_{n}$. Then, as can be readily seen, $\Phi(y)$ and $K_{n}$ itself are the only nonzero $A$ invariant faces of $K_{n}$, both being semi-distinguished $A$-invariant. So condition (b) of Theorem 7.2 is satisfied. For a similar reason, $A^{T}$ also satisfies the corresponding condition. However, $A$ does not satisfy condition (a), because

$$
\operatorname{dim} \mathcal{N}(\rho(A) I-A)=\operatorname{dim} \mathcal{N}(A)=n-\operatorname{rank} A=n-1 \geq 2 .
$$

Conceivably, the geometry of $K$ affects the spectral properties of its cone-preserving maps. We just mention two such results on a rank-one cone-preserving map, whose proofs are not difficult.

Theorem 7.4. Given $\mathbf{0} \neq y \in K$ and $\mathbf{0} \neq z \in K^{*}$ such that $z^{T} y=0$, let $A=y z^{T}$. Then for $\lambda=\rho(A)$, conditions (b), (c) and (d) of Theorem 7.2 are equivalent, and the following is another equivalent condition:

$(a)^{\prime} y$ is an exposed extreme vector of $K$ such that $\Phi(y)=d_{K^{*}}(\Phi(z))$, and the set of all faces of $K$ that contain $y$ forms a chain.

Theorem 7.5. Let $y$ and $z$ be nonzero vectors of $\partial K$ and $\partial K^{*}$ respectively, and let $A=y z^{T}$. The following conditions are equivalent:

(a) $K$ is an A-invariant join-irreducible face of itself.

(b) $y$ belongs to precisely one maximal face of $K$, which also includes $d_{K^{*}}(\Phi(z))$.

If, in addition, the vectors $y$ and $z$ are orthogonal, then the following is another equivalent condition:

(c) $y$ belongs to precisely one maximal face of $K$.

\section{Further examples}

Example 8.1. Let

$$
A=\left[\begin{array}{llll}
1 & 1 & 0 & 0 \\
0 & 1 & 0 & 0 \\
0 & 0 & 1 & 0 \\
0 & 0 & 0 & \alpha
\end{array}\right]
$$


where $0<\alpha<1$. Let $K_{1}, K_{2}$ be convex cones of $\mathbb{R}^{4}$ given by

$$
\begin{aligned}
& K_{1}=\operatorname{pos}\left\{e_{1}, A^{i}\left(e_{2}-e_{4}\right), i=0,1, \cdots\right\}, \\
& K_{2}=\operatorname{pos}\left\{e_{1}, A^{i}\left(e_{2}+e_{3}+e_{4}\right), i=0,1, \cdots\right\},
\end{aligned}
$$

where $e_{1}, \cdots, e_{4}$ are the standard unit vectors of $\mathbb{R}^{4}$. Also let $K=K_{1}+K_{2}$. Clearly $K_{1}, K_{2}$ are closed, pointed cones, and $K$ is a full cone of $\mathbb{R}^{4}$. As can be easily seen, $K_{1} \cap\left(-K_{2}\right)=\{\mathbf{0}\}$. So, by [Roc, Corollary 9.1.2], $K$ is a closed, pointed, full, and hence a proper cone of $\mathbb{R}^{4}$. Since $K_{1}$ and $K_{2}$ are both invariant under $A$, we have $A \in \pi(K)$. Note that the vector $e_{1}$ (and its nonnegative multiples) is the only common vector of $K_{1}$ and $K_{2}$. Except for the vector $e_{1}$, the second component of each vector in $K_{1}$ is always positive and the third component is always zero. On the other hand, the second and the third component of each vector in $K_{2}$, except for the vector $e_{1}$, are always equal and positive. With these observations in mind, it is straightforward to verify that $K_{1}$ and $K_{2}$ are both faces of $K$.

Clearly each of the faces $\Phi\left(e_{1}\right), K_{1}, K_{2}$ and $K$ itself is $A$-invariant.

Indeed, they are precisely all the nonzero $A$-invariant faces of $K$. To see this, let $F$ be any nonzero $A$-invariant face of $K$. If $F$ contains an extreme vector of the form $A^{i}\left(e_{2}-e_{4}\right)$ for some $i \geq 0$, then $F$ will contain all the subsequenct vectors $A^{k}\left(e_{2}-e_{4}\right)$ for $k=i+1, i+2, \cdots$, and hence a relative interior vector of $K_{1}$; thus $F$ includes $K_{1}$. Similarly, $F$ includes $K_{2}$, if $F$ contains an extreme vector of the form $A^{i}\left(e_{2}+e_{3}+e_{4}\right)$ for some $i \geq 0$. But the extreme vectors of $K$ are (up to multiples) precisely the vectors $e_{1}$ and $A^{i}\left(e_{2}-e_{4}\right), A^{i}\left(e_{2}+e_{3}+e_{4}\right), i=0,1,2, \cdots$. With these observations, by considering the possible extreme vectors that $F$ may have, we readily see that $F$ must be one of the faces $\Phi\left(e_{1}\right), K_{1}, K_{2}$ or $K$.

Note that $K$ is $A$-invariant join-reducible, as $K=K_{1} \vee K_{2}$. Also each of the faces $K_{1}, K_{2}$ does not contain in its relative interior a generalized eigenvector of $A$. So, $\Phi\left(e_{1}\right)$ is the only semi-distinguished $A$-invariant face of $K$, and hence, with $\lambda=\rho(A)$, condition (b) of Theorem 7.1 is satisfied trivially. However, as we are going to show, in this case we have $m_{\rho(A)}=2$, so that condition (a) of Theorem 7.1 is not satisfied. Thus, this example shows that in general conditions (a) and (b) of Theorem 7.1 are not equivalent.

Clearly, the vector $e_{2}-e_{4}+A^{2}\left(e_{2}-e_{4}\right)\left(=\left(2,2,0,-\left(1+\alpha^{2}\right)\right)^{T}\right)$ belongs to relint $K_{1}$. Similarly, the vector $e_{2}+e_{3}+e_{4}+A^{2}\left(e_{2}+e_{3}+e_{4}\right)\left(=\left(2,2,2,1+\alpha^{2}\right)^{T}\right)$ belongs to relint $K_{2}$. Hence, their sum, which is $(4,4,2,0)^{T}$, belongs to int $K$. The latter vector is, in fact, also a generalized eigenvector of $A$ corresponding to $\rho(A)$ of order two. This shows that $m_{\rho(A)}=2$, as desired.

Since $\Phi\left(e_{1}\right)$ and $K$ are the only $A$-invariant faces that contain in their relative interiors a generalized eigenvector of $A$, with $\lambda=\rho(A)$, condition (c) of Theorem 7.2 is satisfied. However, condition (b) of Theorem 7.2 is not satisfied (with $\lambda=\rho(A)$ ), as $K$ is not semi-distinguished $A$-invariant. Thus, this example shows that, in general, for the conditions (b), (c) of Theorem 7.2, the implication (c) $\Longrightarrow(b)$ does not hold.

Also note that in this example there does not exist a semi-distinguished $A$ invariant face $F$ such that $\operatorname{sp}_{A}(F)=\operatorname{sp}_{A}(K)$, though $K$ contains in its interior a generalized eigenvector of $A$. So the converse of Remark 6.5 is not true. 
Example 8.2. Take any fixed positive real number $\alpha$ less than 1 , and let

$$
A=\left[\begin{array}{llll}
1 & 1 & 0 & 0 \\
0 & 1 & 0 & 0 \\
0 & 0 & 1 & 0 \\
0 & 0 & 0 & \alpha
\end{array}\right] .
$$

Let $K_{1}, K_{2}$ be the convex cones of $\mathbb{R}^{4}$ given by

$$
\begin{aligned}
& K_{1}=\operatorname{pos}\left\{e_{1}, e_{4}, A^{i}\left(e_{2}-e_{4}\right), i=0,1,2, \cdots\right\}, \\
& K_{2}=\operatorname{pos}\left\{e_{1}, e_{4}, A^{i}\left(e_{2}+e_{3}+e_{4}\right), i=0,1,2, \cdots\right\} .
\end{aligned}
$$

Also, let $K=K_{1}+K_{2}$. Then $K$ is a proper cone of $\mathbb{R}^{4}$ and we have $A \in \pi(K)$. (Note that the cones $K_{1}, K_{2}$ considered here are each obtained from the corresponding cones in Example 8.1 by adjoining the extra extreme ray $\Phi\left(e_{4}\right)$, whereas the matrix $A$ is the same as before.)

Using an argument similar to the one used in Example 8.1, we can readily show that the nonzero $A$-invariant faces of $K$ are precisely $\Phi\left(e_{1}\right), \Phi\left(e_{4}\right), \Phi\left(e_{1}+e_{4}\right), K_{1}, K_{2}$ and $K$ itself. Among these faces, only $\Phi\left(e_{1}\right), K_{1}$ and $K$ have the property that it contains in its relative interior a generalized eigenvector of $A$ corresponding to $\rho(A)$. Furthermore, $\Phi\left(e_{1}\right)$ and $K_{1}$ are the only semi-distinguished $A$-invariant faces associated with $\rho(A)$, and $\operatorname{sp}_{A}\left(K_{1}\right)=\operatorname{sp}_{A}(K)=(1,2)$. Thus, with $\lambda=\rho(A)$, condition (c) of Theorem 6.4 is satisfied. However, condition (a)(i) of Theorem 6.4 is not satisfied, as $K$ cannot be written as the join of semi-distinguished $A$ invariant faces associated with $\rho(A)$. Also note that $K_{2}$ is a nonzero $A$-invariant face associated with $\rho(A)$, but there does not exist a semi-distinguished $A$-invariant face $F$ included in $K_{2}$ such that $\operatorname{sp}_{A}(F)=\operatorname{sp}_{A}\left(K_{2}\right)=(1,2)$. So condition (b)(i) of Theorem 6.4 is also not fulfilled. Thus, this example shows that, in general, condition (c) of Theorem 6.4 does not imply condition (a) nor condition (b) there.

Example 8.3. Let $A=J_{2}(1) \oplus J_{1}(1) \oplus J_{1}(\alpha) \oplus J_{1}(1)$, where $0<\alpha<1$. On $\mathbb{R}^{5}$ define the following cones:

$$
\begin{aligned}
& K_{1}=\operatorname{pos}\left\{e_{1}, A^{i}\left(e_{2}-e_{4}\right), i=0,1,2, \cdots\right\}, \\
& K_{2}=\operatorname{pos}\left\{e_{1}, A^{i}\left(e_{2}+e_{3}+e_{4}\right), i=0,1,2, \cdots\right\}, \\
& K_{3}=\operatorname{pos}\left\{e_{1}, A^{i}\left(e_{2}+e_{4}+e_{5}\right), i=0,1,2, \cdots\right\},
\end{aligned}
$$

and $K=K_{1}+K_{2}+K_{3}$. Clearly $K_{1}, K_{2}$ and $K_{3}$ are each closed, pointed cones. Furthermore, it is easy to verify that

$$
K_{1} \cap\left(-K_{2}\right)=\{\mathbf{0}\} \quad \text { and } \quad\left(K_{1}+K_{2}\right) \cap\left(-K_{3}\right)=\{\mathbf{0}\} .
$$

It follows that $K$ is a proper cone of $\mathbb{R}^{5}$.

It is not difficult to show that $K_{1}, K_{2}$ and $K_{3}$ are all faces of $K$. In addition, we can also show that $K_{1}+K_{2}$ and $K_{1}+K_{3}$ are also faces of $K$. (Whether $K_{2}+K_{3}$ is a face of $K$ is not obvious to us, but we do not need that for our purposes.) Then clearly $K_{1}+K_{2}=K_{1} \vee K_{2}$ and $K_{1}+K_{3}=K_{1} \vee K_{3}$.

Clearly, we have $A \in \pi(K)$. It is not difficult to show that the nonzero $A$ invariant faces of $K$ are precisely $\Phi\left(e_{1}\right), K_{1}, K_{2}, K_{3}, K_{1}+K_{2}, K_{1}+K_{3}, K$, and possibly $K_{2}+K_{3}$. Among these $A$-invariant faces of $K$, only $\Phi\left(e_{1}\right)$ is semi-distinguished $A$-invariant. Note that $K_{1}+K_{2}, K_{1}+K_{3}$ are noncomparable $A$-invariant faces, each containing in its relative interior a generalized eigenvector of $A$ corresponding to $\rho(A)$. So, in this case, with $\lambda=\rho(A)$, condition (d) of Theorem 7.2 is satisfied, 
but not condition (c). This shows that for conditions (c), (d) of Theorem 7.2, in general, we do not have $(\mathrm{d}) \Longrightarrow(\mathrm{c})$.

\section{Open questions}

In trying to improve or better understand Theorem 6.6, we pose the following question.

Question 9.1. Let $K$ be a proper cone whose dual cone $K^{*}$ is a facially exposed cone. Is it true that for any $A \in \pi(K)$, we have the following?

(i) For any nonzero $A$-invariant face $F$ of $K, F$ is semi-distinguished $A$-invariant if and only if $\operatorname{sp}_{A}(F) \succ \operatorname{sp}_{A}(G)$ for all $A$-invariant faces $G$ properly included in $F$.

(ii) There exists in $K$ a generalized eigenvector of $A$ corresponding to $\rho(A)$ of order $\nu_{\rho(A)}(A)$.

(iii) For any $A$-invariant face $F$ which contains in its relative interior a generalized eigenvector of $A$, there exists a semi-distinguished $A$-invariant face $G$ included in $F$ such that $\operatorname{sp}_{A}(G)=\operatorname{sp}_{A}(F)$.

Question 9.2. Let $K$ be a proper cone with the property that the dual cone of each of its faces is a facially exposed cone. Is it true that, for any $A \in \pi(K)$ and any distinguished eigenvalue $\lambda$ of $A$, condition (a) of Theorem 6.4 is always satisfied?

We do not know the answer to Question 9.2 even when $K$ is a perfect cone or is equal to $P(n)$ for some positive integer $n$. If the answer is in the negative, then it will follow that conditions (a) and (b) of Theorem 6.4 are logically independent (cf. Theorem 6.6 and the discussion following Remark 6.5).

\section{REFERENCES}

[Bar1] Barker, G. P., Perfect cones. Linear Algebra Appl. 22, 211-221 (1978) MR 80a:15020

[Bar2] Barker, G. P., Theory of cones. Linear Algebra Appl. 39, 263-291 (1981) MR 83e:15022

[B-P] Berman, A., Plemmons, R. J. Nonnegative Matrices in the Mathematical Sciences. Academic Press, New York, 1979 MR 82b:15013

[B-T] Barker, G. P., Thompson, A., Cones of polynomials. Portugal. Math. 44, 183-197 (1987) MR 88k:52005

[Bir] Birkhoff, G., Lattice Theory. 3rd ed., American Mathematical Society, Providence, R.I., 1966 MR 37:2638

[Dod] Dodds, P. G., Positive compact operators. Quaestiones Math. 18, 21-45 (1995) MR 96c:00020

[G-L-R] Gohberg, L., Lancaster, P., Rodman, L., Invariant Subspaces of Matrices with Applications. John Wiley \& Sons, New York, 1986 MR 88a:15001

[H-S] Hershkowitz, D., Schneider, H., On the generalized nullspace of $M$-matrices and $Z$ matrices. Linear Algebra Appl. 106, 5-23 (1988) MR 90b:15016

[J-V1] Jang, R., Victory, H.D., Jr., On nonnegative solvability of linear integral equations. Linear Algebra Appl. 165, 197-228 (1992) MR 93c:47083

[J-V2] Jang-Lewis, R., Victory, H.D., Jr., On the ideal structure of positive, eventually compact linear operators on Banach lattices. Pacific J. Math. 157, 57-85 (1993) MR 93m:47042

[J-V3] Jang-Lewis, R., Victory, H.D., Jr., On nonnegative solvability of linear operator equations. Integr. Equat. Oper. Th. 18, 88-108 (1994) MR 94m:47075

[MN1] Meyer-Nieberg, P., A partial spectral reduction for positive linear operators. Arch. Math. 45, 34-41 (1985) MR 87a:47065

[MN2] Meyer-Nieberg, P., Banach Lattices, Springer-Verlag, New York, 1991 MR 93f:46025

[Nel] Nelson, Jr., P. The structure of a positive linear integral operator. J. London Math. Soc. (2) 8, 711-718 (1974) MR 50:8169

[Roc] Rockafellar, R. T., Convex Analysis. Princeton Univ. Press, Princeton, NJ, 1970 MR 43:445 
[Rot] Rothblum, U. G., Algebraic eigenspaces of nonnegative matrices. Linear Algebra Appl. 12, 281-292 (1975) MR 53:8100

[Scha] Schaefer, H. H., Banach lattices and positive operators. Springer-Verlag, BerlinHeidelberg-New York, 1974 MR 54:11023

[Schn1] Schneider, H., The elementary divisors associated with 0 of a singular $M$-matrix. Proc. Edinburgh Math. Soc. (2) 10, 108-122 (1956) MR 17:935d

[Schn2] Schneider, H., Geometric conditions for the existence of positive eigenvalues of matrices. Linear Algebra Appl. 38, 253-271 (1981) MR 83f:15012

[Schn3] Schneider, H., The influence of the marked reduced graph of a nonnegative matrix on the Jordan form and on related properties : a survey. Linear Algebra Appl. 84, 161-189 (1986) MR 88b:15010

[Su-T] Sung, C.H., Tam, B. S., A study of projectionally exposed cones. Linear Algebra Appl. 139, 225-252 (1990) MR 91j:52008

[Tam1] Tam, B. S., A note on polyhedral cones. J. Austral. Math. Soc. Ser A 22, 456-461 (1976) MR 55:2962

[Tam2] Tam, B. S., On the duality operator of a convex cone. Linear Algebra Appl. 64, 33-56 (1985) MR 86j:90118

[Tam3] Tam, B. S. On the distinguished eigenvalues of a cone-preserving map. Linear Algebra Appl. 131, 17-37 (1990) MR 91d:15040

[Tam4] Tam, B. S., On semipositive bases for a cone-preserving map, in preparation.

[T-S1] Tam, B. S., Schneider, H., On the core of a cone-preserving map. Trans. Amer. Math. Soc. 343, 479-524 (1994) MR 94h:15011

[T-S2] Tam, B. S., Schneider, H., Linear equations over cones, Collatz-Wielandt numbers and alternating sequences, in preparation.

[T-W] Tam, B. S., Wu, S. F., On the Collatz-Wielandt sets associated with a cone-preserving map. Linear Algebra Appl. 125, 77-95 (1989) MR 90i:15021

[Vic1] Victory, H. D., Jr., On linear integral operators with nonnegative kernels. J. Math. Anal. Appl. 89, 420-441 (1982) MR 84j:45003

[Vic2] Victory, H. D., Jr., The structure of the algebraic eigenspace to the spectral radius of eventually compact, nonnegative integral operators. J. Math. Anal. Appl. 90, 484-516 (1982) MR 84d:47041

[Zer] Zerner, M., Quelques propriétés spectrales des opérateurs positifs. J. Funct. Anal. 72, 381-417 (1987). MR 88i:47020

Department of Mathematics, Tamkang University, Tamsui, Taiwan 25137, ROC

E-mail address: bsm01@mail.tku.edu.tw

Department of Mathematics, University of Wisconsin-Madison, Madison, Wisconsin 53706

E-mail address: hans@math.wisc.edu 